\title{
Co(III)/Zn(II)-catalyzed dearomatization of indoles and coupling with carbenes from ene-yne ketones via intramolecular cyclopropanation
}

\author{
$\mathrm{Na} \mathrm{Li}^{\text {a,b }}$, Junbiao Chang a,*, Lingheng Kong c, Shuangjing Wang a, Dandan Wang a, Miao Chenga , \\ Xingwei Li a,c,\# \\ a Henan Key Laboratory of Organic Functional Molecule and Drug Innovation, School of Chemistry and Chemical Engineering, Henan Normal University, \\ Xinxiang 453007, Henan, China \\ b School of Pharmacy, Xinxiang Medical University, Xinxiang 453003, Henan, China \\ c Dalian Institute of Chemical Physics, Chinese Academy of Sciences, Dalian 116023, Liaoning, China
}

\section{A R T I C L E I N F}

\section{Article history:}

Received 21 June 2018

Accepted 26 July 2018

Published 5 December 2018

\section{Keywords:}

Co(III)/Zn(II) catalysis

Dearomatization

Cyclopropanation

Carbene

Indole

\section{A B S T R A C T}

A straightforward and efficient protocol for dearomatizing indoles is described. The reaction, catalyzed by an inexpensive Co(III)/Zn(II) catalyst, starts from easily accessible $N$-pyrimidinyl indoles and ene-yne ketones. Mild reaction conditions, high diastereoselectivity, a broad substrate scope, effective functional group tolerance, and reasonable to remarkable yields were observed.

(C) 2018, Dalian Institute of Chemical Physics, Chinese Academy of Sciences. Published by Elsevier B.V. All rights reserved.

\section{Introduction}

Indoline skeletons have been considered as characteristic structures owing to their widespread use as key building blocks and chiral auxiliaries in asymmetric synthesis [1]. They are also widely present in alkaloids and other natural products with diverse biological activities [2-9], such as pentopril, which is a potent angiotensin enzyme inhibitor [3], strychnine, which is used as a pesticide [2], and lundurine A, which is effective for overcoming multidrug resistance in vincristine-resistant $\mathrm{KB}$ cells (Fig. 1) [10-12]. Chiral cyclopropanes are important mo- tifs for the diverse functionality and reactivity provided by donor-acceptor substituents, which can be transformed into valuable synthetic intermediates via ring opening or ring expansion [13-20]. A hexacyclic ring system that includes a unique cyclopropyl ring fused to an indoline accompanied by three quaternary carbon stereocenters such as lundurines are attractive targets. Traditional methods to construct cyclopropane-fused indoline derivatives include transition-metal-mediated reactions [21-36], the classic Simmons-Smith reaction and so on [34-41]. However, highly efficient, environmentally friendly, and atom-economic methods

\footnotetext{
* Corresponding author. Tel: +86-411-84379089; E-mail: changjunbiao@zzu.edu.cn

\# Corresponding author. Tel: +86-411-84379089; E-mail: xwli@dicp.ac.cn

This work was supported by the National Natural Science Foundation of China $(21525208,21472186)$ and the research fund from Henan Normal University (5101034011009).

DOI: 10.1016/S1872-2067(18)63154-7 | http://www.sciencedirect.com/science/journal/18722067 | Chin. J. Catal., Vol. 39, No. 12, December 2018
} 


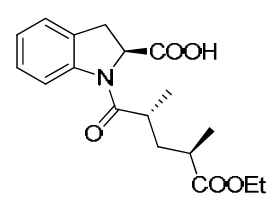

Pentopril

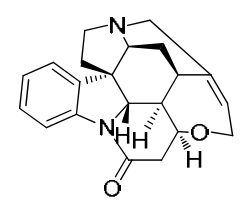

Strychnine

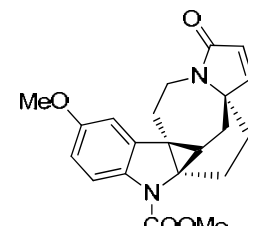

COOMe

Lundurine $\mathrm{A}$
Fig. 1. Some examples of bioactive indolines.

for generating available carbenoids precursors in a catalytic fashion continue to be highly limited [42]. The catalytic generation of M-furylcarbenes derived from carbonyl ene-yne compounds and zinc salts were explored for its versatile character [43-48]. Che et al. [21] reported cobalt(II) porphyrin-catalyzed intramolecular cyclopropanation of $\mathrm{N}$-alkyl indoles/pyrroles with alkyldiazomethanes generated in situ from hydrazones (Scheme 1(a)). Lopez et al. [47] reported the catalytic generation of zinc(II) furylcarbenes derived from carbonyl ene-yne compounds and zinc salts, as well as their reactivity in addition and insertion processes with styrene (Scheme 1(b)). Recently, while collating the data for the present article, Xu et al. [49] explored the access to similar products via $\mathrm{ZnI}_{2}$-catalyzed cyclopropanation of indole with enynone. Notwithstanding the success, the investigation is limited to low functional group tolerance; certain $\mathrm{N}$-substituent of indole failed to generate the desired product (Scheme 1(c)). As well-established and efficient catalysts for various organic transformations, $\mathrm{Cp}^{*} \mathrm{Co}$ (III) complexes have attracted increasing attention owing to their earth-abundance, cost-effectiveness, low toxicity, and unique catalytic reactivity [50-55]. However, the catalytic generation of efficient cyclopropanating intermediates from ene-yne ketone and Co(III)/Zn(II) remains largely underexplored. Herein, we report the intramolecular cyclopropanation of indoles with ene-yne ketones through zinc(II)/Co(III) furylcarbenes. A series of indolines bearing three-dimensional cyclic structures could be obtained with remarkable yields with high to very high diastereoselectivities.

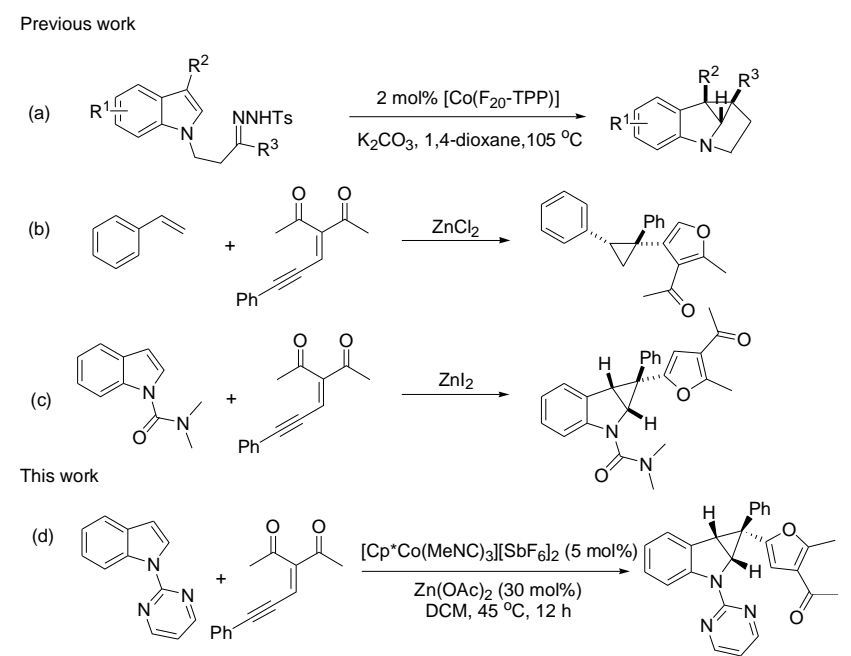

Scheme 1. Cyclopropane reactions of olefins and indoles with metal carbenes.

\section{Experimental}

\subsection{General}

All the chemicals were obtained from commercial sources and were used as-received unless otherwise noted. All the reactions were carried out under $\mathrm{N}_{2}$ atmosphere using standard Schlenk technique. The ${ }^{1} \mathrm{H}$ NMR spectra were recorded on a $400-\mathrm{MHz}$ or $600-\mathrm{MHz}$ NMR spectrometer. The ${ }^{13} \mathrm{C}$ NMR spectra were recorded at $100 \mathrm{MHz}$ or $150 \mathrm{MHz}$. The ${ }^{19} \mathrm{~F}$ NMR spectra were recorded at $565 \mathrm{MHz}$. The chemical shifts were expressed in parts per million $(\delta)$ downfield from the internal standard tetramethylsilane and were reported as s (singlet), d (doublet), $\mathrm{t}$ (triplet), dd (doublet of doublet), dt (doublet of triplet), $\mathrm{m}$ (multiplet), br s (broad singlet), etc. The residual solvent signals were used as references, and the chemical shifts were converted to the TMS scale. High resolution mass spectra were obtained on an Agilent Q-TOF 6540 spectrometer. Column chromatography was performed on silica gel (300-400 mesh) using ethyl acetate (EA)/petroleum ether (PE).

Substrate $1 \mathrm{v}$ was synthesized according to a literature report [56]; the others were prepared following published procedures [57-59]. Compounds $\mathbf{2 a - 2 m}$ were prepared according to a literature report [47].

\subsection{General procedure for synthesizing compounds 3}

$N$-pyrimidinylindole $(0.2 \mathrm{mmol})$, ene-yne ketones $(0.24$ mmol), $\left[\mathrm{Cp}^{*} \mathrm{Co}(\mathrm{MeCN})_{3}\right]\left[\mathrm{SbF}_{6}\right]_{2}$ (5 mol\%), and $\mathrm{Zn}(\mathrm{OAc})_{2}(30$ mol\%) were charged into a Schlenk tube; anhydrous $\mathrm{CH}_{2} \mathrm{Cl}_{2}(2$ $\mathrm{mL}$ ) was added to this mixture under $\mathrm{N}_{2}$ atmosphere. The reaction mixture was stirred at $45^{\circ} \mathrm{C}$ for $12 \mathrm{~h}$. After being cooled to room temperature, the solvent was removed under reduced pressure, and the residue was purified by silica gel chromatography using PE/EA to obtain the product.

\subsection{Spectral data for products}

3aa was obtained according to the general procedure, with $94 \%$ yield, $\mathrm{dr}>20: 1$; pale yellow solid. ${ }^{1} \mathrm{H}$ NMR $(600 \mathrm{MHz}$, $\left.\mathrm{CDCl}_{3}\right) \delta 8.54(\mathrm{~d}, J=4.7 \mathrm{~Hz}, 2 \mathrm{H}), 8.17(\mathrm{~d}, J=8.2 \mathrm{~Hz}, 1 \mathrm{H}), 7.43(\mathrm{~d}$, $J=7.3 \mathrm{~Hz}, 1 \mathrm{H}), 7.34(\mathrm{~m}, 2 \mathrm{H}), 7.29-7.21(\mathrm{~m}, 3 \mathrm{H}), 7.14(\mathrm{t}, J=7.8$ $\mathrm{Hz}, 1 \mathrm{H}), 6.94(\mathrm{t}, J=7.4 \mathrm{~Hz}, 1 \mathrm{H}), 6.78(\mathrm{t}, J=4.7 \mathrm{~Hz}, 1 \mathrm{H}), 5.75(\mathrm{~s}$, $1 \mathrm{H}), 5.20(\mathrm{~d}, J=6.5 \mathrm{~Hz}, 1 \mathrm{H}), 3.51(\mathrm{~d}, J=6.5 \mathrm{~Hz}, 1 \mathrm{H}), 2.15(\mathrm{~s}, 3 \mathrm{H})$, 2.01 (s, 3H). ${ }^{13} \mathrm{C}$ NMR (151 MHz, $\left.\mathrm{CDCl}_{3}\right) \delta 194.0,159.8,157.7$, 147.8, 143.8, 142.0, 130.6, 128.7, 127.6, 126.7, 126.6, 124.8, $121.5121 .3,115.6,112.7,111.1,51.9,35.9,28.8,27.2,14.0$. HRMS calculated for $\mathrm{C}_{26} \mathrm{H}_{22} \mathrm{~N}_{3} \mathrm{O}_{2}{ }^{+}(\mathrm{M}+\mathrm{H})^{+}$: 408.1707; observed: 408.408.1709.

$3 \mathbf{b a}$ was obtained according to the general procedure, with $62 \%$ yield, $\mathrm{dr}=15: 1$; pale yellow solid. ${ }^{1} \mathrm{H}$ NMR $(400 \mathrm{MHz}$, $\left.\mathrm{CDCl}_{3}\right) \delta 8.54(\mathrm{~d}, J=4.8 \mathrm{~Hz}, 2 \mathrm{H}), 7.80(\mathrm{~d}, J=8.2 \mathrm{~Hz}, 1 \mathrm{H})$, 7.37-7.31 (m, 2H), 7.29-7.22 (m, 3H), 7.09 (t, $J=8.2 \mathrm{~Hz}, 1 \mathrm{H})$, $6.78(\mathrm{t}, J=4.8 \mathrm{~Hz}, 1 \mathrm{H}), 6.52(\mathrm{~d}, J=8.2 \mathrm{~Hz}, 1 \mathrm{H}), 5.80(\mathrm{~s}, 1 \mathrm{H}), 5.14$ (d, $J=6.6 \mathrm{~Hz}, 1 \mathrm{H}), 3.91(\mathrm{~s}, 3 \mathrm{H}), 3.62$ (d, $J=6.6 \mathrm{~Hz}, 1 \mathrm{H}), 2.17(\mathrm{~s}$, 3H), $2.04(\mathrm{~s}, 3 \mathrm{H}) \cdot{ }^{13} \mathrm{C}$ NMR $\left(151 \mathrm{MHz}, \mathrm{CDCl}_{3}\right) \delta 194.0,159.8$, 157.7 (d, $J=7.4 \mathrm{~Hz}$ ), 156.6, 148.1, 145.2, 142.1, 128.8, 128.6, 
126.8, 126.5, 121.3, 118.5, 112.7, 110.9, 108.9, 104.4, 55.8, 52.3, 32.9 , 28.8, 26.8, 14.1. HRMS calculated for $\mathrm{C}_{27} \mathrm{H}_{24} \mathrm{~N}_{3} \mathrm{O}_{3}{ }^{+}(\mathrm{M}+$ $\mathrm{H}^{+}$: 438.1812; observed: 438.1819 .

3ca was obtained according to the general procedure, with $92 \%$ yield, $\mathrm{dr}=7: 1$; pale yellow solid. ${ }^{1} \mathrm{H}$ NMR $(400 \mathrm{MHz}$, $\left.\mathrm{CDCl}_{3}\right) \delta 8.53(\mathrm{~d}, J=4.8 \mathrm{~Hz}, 2 \mathrm{H}), 8.34(\mathrm{~d}, J=8.0 \mathrm{~Hz}, 1 \mathrm{H}), 7.53(\mathrm{~d}$, $J=7.0 \mathrm{~Hz}, 1 \mathrm{H}), 7.47(\mathrm{dd}, J=8.1,2.0 \mathrm{~Hz}, 2 \mathrm{H}), 7.31(\mathrm{t}, J=7.6 \mathrm{~Hz}$, 2H), 7.23-7.18 (m, 1H), $7.14(\mathrm{t}, J=8.0 \mathrm{~Hz}, 1 \mathrm{H}), 6.78(\mathrm{t}, J=4.8$ $\mathrm{Hz}, 1 \mathrm{H}), 5.63(\mathrm{~s}, 1 \mathrm{H}), 5.12(\mathrm{~d}, J=6.4 \mathrm{~Hz}, 1 \mathrm{H}), 4.15(\mathrm{~d}, J=6.4 \mathrm{~Hz}$, $1 \mathrm{H}), 3.95$ (s, 3H), 2.03 (s, 3H), 1.93 (s, 3H). ${ }^{13} \mathrm{C}$ NMR (151 MHz, $\left.\mathrm{CDCl}_{3}\right) \delta 193.9,167.1,159.8,157.6,148.5,144.9,141.3,132.9$, $128.7,128.2,127.5,127.0,126.8,122.8,121.3,119.5,112.9$, 110.2, 52.1, 51.8, 35.0, 28.8, 27.8, 14.0. HRMS calculated for $\mathrm{C}_{28} \mathrm{H}_{24} \mathrm{~N}_{3} \mathrm{O}_{4}+(\mathrm{M}+\mathrm{H})^{+}:$466.1761; observed: 466.1767.

3da was obtained according to the general procedure, with $44 \%$ yield, $\mathrm{dr}=5: 1$; pale yellow solid. ${ }^{1} \mathrm{H}$ NMR $(600 \mathrm{MHz}$, $\left.\mathrm{CDCl}_{3}\right) \delta 8.56(\mathrm{~d}, J=4.7 \mathrm{~Hz}, 2 \mathrm{H}), 8.00(\mathrm{~d}, J=8.2 \mathrm{~Hz}, 1 \mathrm{H}), 7.36(\mathrm{t}, J$ $=7.6 \mathrm{~Hz}, 2 \mathrm{H}), 7.31(\mathrm{~d}, J=7.1 \mathrm{~Hz}, 2 \mathrm{H}), 7.29-7.24(\mathrm{~m}, 1 \mathrm{H}), 7.04(\mathrm{t}$, $J=7.9 \mathrm{~Hz}, 1 \mathrm{H}), 6.79(\mathrm{t}, J=4.7 \mathrm{~Hz}, 1 \mathrm{H}), 6.76(\mathrm{~d}, J=7.5 \mathrm{~Hz}, 1 \mathrm{H})$, $5.71(\mathrm{~s}, 1 \mathrm{H}), 5.15(\mathrm{~d}, J=6.7 \mathrm{~Hz}, 1 \mathrm{H}), 3.47(\mathrm{~d}, J=6.7 \mathrm{~Hz}, 1 \mathrm{H}), 2.51$ (s, 3H), 2.16 (s, 3H), 2.02 (s, 3H). ${ }^{13} \mathrm{C}$ NMR (151 MHz, $\left.\mathrm{CDCl}_{3}\right) \delta$ 193.9, 159.9, 157.7, 147.99 (s), 143.6, 142.1, 134.3, 129.5, 128.7, 127.7, 127.3, 126.6, 122.5, 121.3, 113.1, 112.6, 110.6, 51.9, 34.3, 28.8, 27.2, 18.8, 14.0. HRMS calculated for $\mathrm{C}_{27} \mathrm{H}_{24} \mathrm{~N}_{3} \mathrm{O}_{2}{ }^{+}(\mathrm{M}+\mathrm{H})^{+}:$: 422.1863; observed: 422.1866 .

3ea was obtained according to the general procedure, with $80 \%$ yield, $\mathrm{dr}=16: 1$; pale yellow solid. ${ }^{1} \mathrm{H}$ NMR $(400 \mathrm{MHz}$, $\left.\mathrm{CDCl}_{3}\right) \delta 8.58(\mathrm{~d}, J=4.8 \mathrm{~Hz}, 2 \mathrm{H}), 8.07(\mathrm{~d}, J=8.2 \mathrm{~Hz}, 1 \mathrm{H})$, 7.44-7.33 (m, 4H), 7.31-7.25 (m, 1H), $7.07(\mathrm{t}, J=8.1 \mathrm{~Hz}, 1 \mathrm{H})$, $6.94(\mathrm{~d}, J=8.0,1 \mathrm{H}), 6.85(\mathrm{t}, J=4.8 \mathrm{~Hz}, 1 \mathrm{H}), 5.81(\mathrm{~s}, 1 \mathrm{H}), 5.20(\mathrm{~d}$, $J=6.6 \mathrm{~Hz}, 1 \mathrm{H}), 3.66(\mathrm{~d}, J=6.6 \mathrm{~Hz}, 1 \mathrm{H}), 2.16(\mathrm{~s}, 3 \mathrm{H}), 2.05(\mathrm{~s}, 3 \mathrm{H})$. ${ }^{13} \mathrm{C}$ NMR $\left(151 \mathrm{MHz}, \mathrm{CDCl}_{3}\right) \delta 193.9,159.6,157.9,157.7,147.9$, $145.0,141.3,130.6,129.2,128.8,128.7,127.4,126.9,121.3$, 113.8, 113, 110.7, 51.6, 33.9, 28.8, 27.2, 14.0. HRMS calculated for $\mathrm{C}_{26} \mathrm{H}_{21} \mathrm{ClN}_{3} \mathrm{O}_{2}+(\mathrm{M}+\mathrm{H})^{+}:$442.1317; observed: 442.1316 .

3fa was obtained according to the general procedure, with $66 \%$ yield, $\mathrm{dr}=11: 1$; pale yellow solid. ${ }^{1} \mathrm{H}$ NMR $(400 \mathrm{MHz}$, $\left.\mathrm{CDCl}_{3}\right) \delta 8.51(\mathrm{~d}, J=4.8 \mathrm{~Hz}, 2 \mathrm{H}), 7.88(\mathrm{~d}, J=8.2 \mathrm{~Hz}, 1 \mathrm{H})$, 7.33-7.25 (m, 4H), 7.24-7.16 (m, 1H), $7.02(\mathrm{td}, J=8.3,5.9 \mathrm{~Hz}$, $1 \mathrm{H}), 6.78(\mathrm{t}, J=4.8 \mathrm{~Hz}, 1 \mathrm{H}), 6.60(\mathrm{t}, J=8.5 \mathrm{~Hz}, 1 \mathrm{H}), 5.76(\mathrm{~s}, 1 \mathrm{H})$, $5.15(\mathrm{~d}, J=6.6 \mathrm{~Hz}, 1 \mathrm{H}), 3.58(\mathrm{~d}, J=6.6 \mathrm{~Hz}, 1 \mathrm{H}), 2.10(\mathrm{~s}, 3 \mathrm{H}), 1.97$ (s, 3H). ${ }^{13} \mathrm{C}$ NMR $\left(151 \mathrm{MHz}, \mathrm{CDCl}_{3}\right) \delta 194.1,160.6,159.6,158.9$, $157.9157 .7,147.8,146.1$ (d, $J=7.9 \mathrm{~Hz}), 141.3,129.1$ (d, $J=8.1$ $\mathrm{Hz}), 128.7,127.2,126.9,121.3,117.3(\mathrm{~d}, J=21.5 \mathrm{~Hz}$ ), 113.1, 111.5, 110.8, 108.2 (d, $J=19.7 \mathrm{~Hz}$ ), 52.1, 31.4, 28.8, 26.9, 14.0. ${ }^{19} \mathrm{~F}$ NMR $\left(565 \mathrm{MHz}, \mathrm{CDCl}_{3}\right) \delta-121.76(1 \mathrm{~F})$. HRMS calculated for $\mathrm{C}_{26} \mathrm{H}_{21} \mathrm{FN}_{3} \mathrm{O}_{2}+(\mathrm{M}+\mathrm{H})^{+}:$426.1612; observed: 426.1613 .

3ga was obtained according to the general procedure, with $94 \%$ yield, dr > 20:1; pale yellow solid. ${ }^{1} \mathrm{H}$ NMR $(600 \mathrm{MHz}$, $\left.\mathrm{CDCl}_{3}\right) \delta 8.44(\mathrm{~d}, J=4.7 \mathrm{~Hz}, 2 \mathrm{H}), 7.94(\mathrm{dd}, J=8.2,2.6 \mathrm{~Hz}, 1 \mathrm{H})$, $7.24(\mathrm{t}, J=7.7 \mathrm{~Hz}, 2 \mathrm{H}), 7.15(\mathrm{dd}, J=14.7,7.3 \mathrm{~Hz}, 4 \mathrm{H}), 6.85(\mathrm{~d}, J=$ $8.2 \mathrm{~Hz}, 1 \mathrm{H}), 6.66(\mathrm{t}, J=4.7 \mathrm{~Hz}, 1 \mathrm{H}), 5.67(\mathrm{~s}, 1 \mathrm{H}), 5.07$ (d, $J=6.5$ $\mathrm{Hz}, 1 \mathrm{H}), 3.37$ (d, J = 6.5 Hz, 1H), 2.23 (s, 3H), 2.08 (s, 3H), 1.94 (s, 3H). ${ }^{13} \mathrm{C}$ NMR $\left(151 \mathrm{MHz}, \mathrm{CDCl}_{3}\right) \delta 194.0,159.8,157.7$ (d, $J=$ $10.3 \mathrm{~Hz}), 147.9,142.1$ 141.6, 131.0, 130.6, 128.6, 128.0, 126.7, $126.5,125.4,121.3,115.4,112.3,111.0,52.1,35.9,28.8,27.5$, 20.9, 14.0. HRMS calculated for $\mathrm{C}_{27} \mathrm{H}_{24} \mathrm{~N}_{3} \mathrm{O}_{2}{ }^{+}(\mathrm{M}+\mathrm{H})^{+}$:
422.1863; observed: 422.1866 .

3ha was obtained according to the general procedure, with $84 \%$ yield, $\mathrm{dr}=4: 1$; pale yellow solid. ${ }^{1} \mathrm{H}$ NMR $(400 \mathrm{MHz}$, $\left.\mathrm{CDCl}_{3}\right) \delta 8.53(\mathrm{~d}, J=4.8 \mathrm{~Hz}, 2 \mathrm{H}), 8.07(\mathrm{~d}, J=8.9 \mathrm{~Hz}, 1 \mathrm{H}), 7.35(\mathrm{~m}$, $2 \mathrm{H}), 7.29-7.22(\mathrm{~m}, 4 \mathrm{H}), 7.04(\mathrm{~d}, J=2.7 \mathrm{~Hz}, 1 \mathrm{H}), 6.76(\mathrm{t}, J=4.8$ $\mathrm{Hz}, 1 \mathrm{H}), 6.70$ (dd, $J=8.9,2.7 \mathrm{~Hz}, 1 \mathrm{H}), 5.79(\mathrm{~s}, 1 \mathrm{H}), 5.19$ (d, $J=6.5$ Hz, 1H), 3.80 (s, 3H), 3.49 (d, $J=6.5 \mathrm{~Hz}, 1 \mathrm{H}), 2.18$ (s, 3H), 2.05 (s, 3H). ${ }^{13} \mathrm{C} \mathrm{NMR}\left(151 \mathrm{MHz}, \mathrm{CDCl}_{3}\right) \delta 194.4,158.0,157.9,157.4$, $155.4,140.2,130.9,130.2,128.9,128.7,127.1,125.2,122.1$, 120.4, 117.3, 115.8, 112.5, 108.3, 102.2, 55.7, 44.8, 29.2, 14.6. HRMS calculated for $\mathrm{C}_{27} \mathrm{H}_{24} \mathrm{~N}_{3} \mathrm{O}_{3}{ }^{+}(\mathrm{M}+\mathrm{H})^{+}$: 438.1812; observed: 438.1813.

3ia was obtained according to the general procedure, with $77 \%$ yield, $\mathrm{dr}=9: 1$; pale yellow solid. ${ }^{1} \mathrm{H}$ NMR $(600 \mathrm{MHz}$, $\left.\mathrm{CDCl}_{3}\right) \delta 8.48(\mathrm{~d}, J=3.4 \mathrm{~Hz}, 2 \mathrm{H}), 8.11(\mathrm{~d}, J=5.1 \mathrm{~Hz}, 1 \mathrm{H}), 8.01(\mathrm{~s}$, $1 \mathrm{H}), 7.77(\mathrm{~d}, J=1.5 \mathrm{~Hz}, 1 \mathrm{H}), 7.34-7.02(\mathrm{~m}, 5 \mathrm{H}), 6.76(\mathrm{~s}, 1 \mathrm{H})$, $5.68(\mathrm{~s}, 1 \mathrm{H}), 5.11(\mathrm{~d}, J=6.5 \mathrm{~Hz}, 1 \mathrm{H}), 3.79(\mathrm{~s}, 3 \mathrm{H}), 3.41(\mathrm{~d}, J=6.5$ $\mathrm{Hz}, 1 \mathrm{H}), 2.04$ (s, 3H), 1.91 (s, 3H). $\left.{ }^{13} \mathrm{C} \mathrm{NMR} \mathrm{(151} \mathrm{MHz,} \mathrm{CDCl}_{3}\right) \delta$ 193.8, 167.0, 159.5, 157.8, 147.6, 141.32 (s), 130.7, 130.1, 128.7, 127.1, 126.9, 126.2, 123.0, 121.3, 114.8, 113.6, 111.0, 52.1, 51.9, 35.0, 28.8, 27.1, 14.1. HRMS calculated for $\mathrm{C}_{28} \mathrm{H}_{24} \mathrm{~N}_{3} \mathrm{O}_{4}+(\mathrm{M}+\mathrm{H})^{+}:$466.1761; observed: 466.1763 .

3ja was obtained according to the general procedure, with $62 \%$ yield, $\mathrm{dr}>20: 1$; pale yellow solid. ${ }^{1} \mathrm{H}$ NMR $(400 \mathrm{MHz}$, $\left.\mathrm{CDCl}_{3}\right) \delta 8.47$ (d, $\left.J=4.8 \mathrm{~Hz}, 2 \mathrm{H}\right), 8.06(\mathrm{dd}, J=9.0,4.8 \mathrm{~Hz}, 1 \mathrm{H}$ ), 7.30-7.25 (m, 2H), 7.26-7.15 (m, 3H), 7.08 (dd, $J=8.1,2.7 \mathrm{~Hz}$, $1 \mathrm{H}), 6.76$ (ddd, $J=9.6,8.2,3.7 \mathrm{~Hz}, 2 \mathrm{H}$ ), $5.72(\mathrm{~s}, 1 \mathrm{H}), 5.15$ (d, $J=$ $6.5 \mathrm{~Hz}, 1 \mathrm{H}$ ), 3.42 (d, $J=6.5 \mathrm{~Hz}, 1 \mathrm{H}), 2.11(\mathrm{~s}, 3 \mathrm{H}), 1.98(\mathrm{~s}, 3 \mathrm{H}) .{ }^{13} \mathrm{C}$ NMR $\left(151 \mathrm{MHz}, \mathrm{CDCl}_{3}\right) \delta 193.9,159.6,158.9,157.9,157.7$, $157.3,147.6,141.6,139.9,132.1(\mathrm{~d}, J=8.8 \mathrm{~Hz}), 128.7,126.8$ (d, $J=2.9 \mathrm{~Hz}), 121.3,116.3(\mathrm{~d}, J=8.0 \mathrm{~Hz}), 113.8(\mathrm{~d}, J=22.7 \mathrm{~Hz})$, 112.7, 111.9 (d, $J=24.3 \mathrm{~Hz}$ ), 111.0, 52.3, 35.5, 28.8, 27.4, 14.0. ${ }^{19} \mathrm{~F}$ NMR (376 MHz, $\mathrm{CDCl}_{3}$ ) $\delta-122.30$ (1F). HRMS calculated for $\mathrm{C}_{26} \mathrm{H}_{21} \mathrm{FN}_{3} \mathrm{O}_{2}+(\mathrm{M}+\mathrm{H})^{+}:$426.1612; observed: 426.1618 .

3ka was obtained according to the general procedure, with $64 \%$ yield, $\mathrm{dr}=16: 1$; pale yellow solid. ${ }^{1} \mathrm{H}$ NMR $(400 \mathrm{MHz}$, $\left.\mathrm{CDCl}_{3}\right) \delta 8.48(\mathrm{~d}, J=4.8 \mathrm{~Hz}, 2 \mathrm{H}), 7.99(\mathrm{~d}, J=8.7 \mathrm{~Hz}, 1 \mathrm{H}), 7.47(\mathrm{~d}$, $J=2.1 \mathrm{~Hz}, 1 \mathrm{H}), 7.38-7.23(\mathrm{~m}, 2 \mathrm{H}), 7.22-7.13(\mathrm{~m}, 4 \mathrm{H}), 6.75$ (s, $1 \mathrm{H}), 5.72(\mathrm{~s}, 1 \mathrm{H}), 5.12(\mathrm{~d}, J=6.5 \mathrm{~Hz}, 1 \mathrm{H}), 3.40(\mathrm{~d}, J=6.5 \mathrm{~Hz}, 1 \mathrm{H})$, $2.11(\mathrm{~s}, 3 \mathrm{H}), 1.98(\mathrm{~s}, 3 \mathrm{H}) .{ }^{13} \mathrm{C}$ NMR (151 MHz, $\left.\mathrm{CDCl}_{3}\right) \delta 193.9$, 159.6, $157.9157 .7,147.5,142.8,141.4,132.8,130.3,128.7$, 127.7, 126.8, 126.8, 121.3, 117.0, 113.7, 113.0, 111.1, 52.0, 35.1, 28.8, 27.2, 14.0. HRMS calculated for $\mathrm{C}_{26} \mathrm{H}_{21} \mathrm{BrN}_{3} \mathrm{O}_{2}+(\mathrm{M}+\mathrm{H})^{+}$: 486.0812; observed: 486.0810 .

3la was obtained according to the general procedure, with $66 \%$ yield, $\mathrm{dr}=16: 1$; pale yellow solid. ${ }^{1} \mathrm{H}$ NMR $(400 \mathrm{MHz}$, $\left.\mathrm{CDCl}_{3}\right) \delta 8.52(\mathrm{~d}, J=4.8 \mathrm{~Hz}, 2 \mathrm{H}), 8.18(\mathrm{~d}, J=8.6 \mathrm{~Hz}, 1 \mathrm{H}), 7.61(\mathrm{~d}$, $J=1.3 \mathrm{~Hz}, 1 \mathrm{H}), 7.35-7.26(\mathrm{~m}, 3 \mathrm{H}), 7.24-7.16(\mathrm{~m}, 3 \mathrm{H}), 6.81(\mathrm{t}, J=$ $4.8 \mathrm{~Hz}, 1 \mathrm{H}), 5.69$ (s, $1 \mathrm{H}), 5.19$ (d, $J=6.5 \mathrm{~Hz}, 1 \mathrm{H}), 3.48$ (d, $J=6.5$ $\mathrm{Hz}, 1 \mathrm{H}), 2.07$ (s, 3H), $1.94(\mathrm{~s}, 3 \mathrm{H}) \cdot{ }^{13} \mathrm{C} \mathrm{NMR}\left(151 \mathrm{MHz}, \mathrm{CDCl}_{3}\right) \delta$ 193.8, 159.5, 157.9, 157.8, 147.4, 146.2, 141.2, 131.2, 128.8, $126.9(\mathrm{~d}, J=4.1 \mathrm{~Hz}), 125.0(\mathrm{~d}, J=3.8 \mathrm{~Hz}), 121.8(\mathrm{~d}, J=3.7 \mathrm{~Hz}$ ), 121.3, 115.2, 113.6, 111.3, 52.0, 35.0, 28.7, 26.9, 13.9. ${ }^{19} \mathrm{~F}$ NMR (376 $\left.\mathrm{MHz}, \mathrm{CDCl}_{3}\right) \delta-61.24$ (3F). HRMS calculated for $\mathrm{C}_{27} \mathrm{H}_{21} \mathrm{~F}_{3} \mathrm{~N}_{3} \mathrm{O}_{2}+(\mathrm{M}+\mathrm{H})^{+}:$: 476.1580; observed: 476.1583.

$3 \mathrm{ma}$ was obtained according to the general procedure, with $58 \%$ yield, $\mathrm{dr}=7: 1$; pale yellow solid. ${ }^{1} \mathrm{H}$ NMR $(400 \mathrm{MHz}$, 
$\left.\mathrm{CDCl}_{3}\right) \delta 8.52(\mathrm{~d}, J=4.8 \mathrm{~Hz}, 2 \mathrm{H}), 8.07(\mathrm{~d}, J=8.9 \mathrm{~Hz}, 1 \mathrm{H}), 7.43(\mathrm{~m}$, 2H), $7.34(\mathrm{~m}, 5 \mathrm{H}), 7.24(\mathrm{~m}, 3 \mathrm{H}), 7.10(\mathrm{~d}, J=2.6 \mathrm{~Hz}, 1 \mathrm{H}), 6.76(\mathrm{dt}$, $J=8.1,3.7 \mathrm{~Hz}, 2 \mathrm{H}), 5.77(\mathrm{~s}, 1 \mathrm{H}), 5.17(\mathrm{~d}, J=6.5 \mathrm{~Hz}, 1 \mathrm{H}), 5.06(\mathrm{~s}$, 2H), 3.47 (d, $J=6.5 \mathrm{~Hz}, 1 \mathrm{H}), 2.17(\mathrm{~s}, 3 \mathrm{H}), 2.03(\mathrm{~s}, 3 \mathrm{H}) .{ }^{13} \mathrm{C}$ NMR (151 MHz, $\left.\mathrm{CDCl}_{3}\right) \delta 194.0,159.7,157.7,154.0,147.8,141.9$, 138.0, 137.3, $131.8128 .7,128.6,127.9,127.4,126.7,126.6$, 121.3, 116.2, 113.8, 112.2, 110.9, 70.7, 52.3, 36.0, 28.9, 27.7, 14.1. HRMS calculated for $\mathrm{C}_{32} \mathrm{H}_{26} \mathrm{~N}_{3} \mathrm{O}_{3}+(\mathrm{M}+\mathrm{H})^{+}$: 500.1969 ; observed: 500.1974 .

3pa was obtained according to the general procedure, with $72 \%$ yield, $\mathrm{dr}=18: 1$; pale yellow solid. ${ }^{1} \mathrm{H}$ NMR $(400 \mathrm{MHz}$, $\left.\mathrm{CDCl}_{3}\right) \delta 8.48(\mathrm{~d}, J=4.8 \mathrm{~Hz}, 2 \mathrm{H}), 7.93(\mathrm{~s}, 1 \mathrm{H}), 7.30-7.21(\mathrm{~m}, 3 \mathrm{H})$, $7.17(\mathrm{dd}, J=6.4,2.0 \mathrm{~Hz}, 3 \mathrm{H}), 6.74-6.66(\mathrm{~m}, 2 \mathrm{H}), 5.68(\mathrm{~s}, 1 \mathrm{H})$, $5.10(\mathrm{~d}, J=6.6 \mathrm{~Hz}, 1 \mathrm{H}), 3.39$ (d, $J=6.6 \mathrm{~Hz}, 1 \mathrm{H}), 2.25(\mathrm{~s}, 3 \mathrm{H}), 2.10$ (s, 3H), 1.96 (s, 3H). ${ }^{13} \mathrm{C}$ NMR (151 MHz, CDCl 3 ) $\delta 194.2,159.8$, 157.7, 157.6, 148.0, 144.0, 142.2, 137.4, 128.6, 127.8, 126.7, $126.5,124.4,122.3,121.3,116.2,112.5,111.1,52.3,35.7,28.8$, 27.4, 21.9, 14.1. HRMS calculated for $\mathrm{C}_{27} \mathrm{H}_{24} \mathrm{~N}_{3} \mathrm{O}_{2}{ }^{+}(\mathrm{M}+\mathrm{H})^{+}$: 422.1863; observed: 422.1861 .

3qa was obtained according to the general procedure, with $40 \%$ yield, $\mathrm{dr}=5: 1$; pale yellow solid. ${ }^{1} \mathrm{H}$ NMR $(400 \mathrm{MHz}$, $\left.\mathrm{CDCl}_{3}\right) \delta 8.56(\mathrm{~d}, J=4.8 \mathrm{~Hz}, 2 \mathrm{H}), 7.88(\mathrm{~d}, J=2.4 \mathrm{~Hz}, 1 \mathrm{H})$, $7.37-7.28(\mathrm{~m}, 3 \mathrm{H}), 7.26(\mathrm{~s}, 2 \mathrm{H}), 7.24(\mathrm{~s}, 1 \mathrm{H}), 6.81(\mathrm{t}, J=4.8 \mathrm{~Hz}$, $1 \mathrm{H}), 6.50(\mathrm{dd}, J=8.2,2.4 \mathrm{~Hz}, 1 \mathrm{H}), 5.77(\mathrm{~s}, 1 \mathrm{H}), 5.18(\mathrm{~d}, J=6.7 \mathrm{~Hz}$, 1H), 3.79 (s, 3H), $3.46(\mathrm{~d}, J=6.7 \mathrm{~Hz}, 1 \mathrm{H}), 2.18(\mathrm{~s}, 3 \mathrm{H}), 2.05(\mathrm{~s}$, 3H). ${ }^{13} \mathrm{C}$ NMR (151 MHz, $\left.\mathrm{CDCl}_{3}\right) \delta 194.1,159.7,157.7,148.0$, $145.1,142.1,128.6,126.6,124.9,123.1,121.3,112 ., 111.0$, 106.8, 102.6, 55.5, 52.6, 35.3, 28.8, 27.5, 14.1. HRMS calculated for $\mathrm{C}_{27} \mathrm{H}_{24} \mathrm{~N}_{3} \mathrm{O}_{3}+(\mathrm{M}+\mathrm{H})^{+}$: 438.1812; observed: 438.1813 .

3ra was obtained according to the general procedure, with $77 \%$ yield, $\mathrm{dr}=9: 1$; pale yellow solid. ${ }^{1} \mathrm{H}$ NMR $(400 \mathrm{MHz}$, $\left.\mathrm{CDCl}_{3}\right) \delta 8.74(\mathrm{~s}, 1 \mathrm{H}), 8.55(\mathrm{~d}, J=4.8 \mathrm{~Hz}, 2 \mathrm{H}), 7.62$ (dd, $J=7.8$, $1.5 \mathrm{~Hz}, 1 \mathrm{H}), 7.43(\mathrm{~d}, J=7.8 \mathrm{~Hz}, 1 \mathrm{H}), 7.33-7.26(\mathrm{~m}, 2 \mathrm{H})$, 7.24-7.20 (m, 3H), $6.79(\mathrm{t}, J=4.8 \mathrm{~Hz}, 1 \mathrm{H}), 5.72(\mathrm{~s}, 1 \mathrm{H}), 5.20(\mathrm{~d}, J$ $=6.4 \mathrm{~Hz}, 1 \mathrm{H}), 3.84(\mathrm{~s}, 3 \mathrm{H}), 3.49(\mathrm{~d}, J=6.4 \mathrm{~Hz}, 1 \mathrm{H}), 2.09(\mathrm{~s}, 3 \mathrm{H})$, $1.96(\mathrm{~s}, 3 \mathrm{H}) .{ }^{13} \mathrm{C}$ NMR $\left(151 \mathrm{MHz}, \mathrm{CDCl}_{3}\right) \delta 193.8,167.0,159.5$, 157.8, 147.6, 141.3, 130.7, 130.1, 128.7, 127.1, 126.9, 126.2, 123.0, 121.3, 114.8, 113.6, 111.0, 52.1, 51.9, 35.0, 28.8, 27.1, 14.0. HRMS calculated for $\mathrm{C}_{28} \mathrm{H}_{24} \mathrm{~N}_{3} \mathrm{O}_{4}+(\mathrm{M}+\mathrm{H})^{+}$: 466.1761; observed: 466.1764 .

3sa was obtained according to the general procedure, with $62 \%$ yield, $\mathrm{dr}>20: 1$; pale yellow solid. ${ }^{1} \mathrm{H}$ NMR $(400 \mathrm{MHz}$, $\left.\mathrm{CDCl}_{3}\right) \delta 8.50(\mathrm{~d}, J=4.8 \mathrm{~Hz}, 2 \mathrm{H}), 8.32(\mathrm{~d}, J=1.7 \mathrm{~Hz}, 1 \mathrm{H}), 7.27(\mathrm{~m}$, 2H), $7.18(\mathrm{~m}, 4 \mathrm{H}), 7.00(\mathrm{dd}, J=7.9,1.8 \mathrm{~Hz}, 1 \mathrm{H}), 6.77(\mathrm{t}, J=4.8$ $\mathrm{Hz}, 1 \mathrm{H}), 5.70$ (s, 1H), 5.12 (d, $J=6.6 \mathrm{~Hz}, 1 \mathrm{H}), 3.39$ (d, $J=6.6 \mathrm{~Hz}$, 1H), 2.11 (s, 3H), 1.99 (s, 3H). ${ }^{13} \mathrm{C}$ NMR (151 MHz, CDCl 3$) \delta$ 193.9, 159.5, 157.9, 157.7, 147.6, 144.9, 141.6, 129.7, 128.7, 126.8, 125.8, 124.3, 121.4, 121.1, 118.7, 113.2, 111.1, 52.2, 35.3, 28.9, 27.09, 14.1. HRMS calculated for $\mathrm{C}_{26} \mathrm{H}_{21} \mathrm{BrN}_{3} \mathrm{O}_{2}+(\mathrm{M}+\mathrm{H})^{+}$: 486.0812; observed: 486.0819 .

3ta was obtained according to the general procedure, with $84 \%$ yield, $\mathrm{dr}=16: 1$; pale yellow solid. ${ }^{1} \mathrm{H}$ NMR $(400 \mathrm{MHz}$, $\left.\mathrm{CDCl}_{3}\right) \delta 8.48(\mathrm{~d}, J=4.8 \mathrm{~Hz}, 2 \mathrm{H}), 7.90(\mathrm{dd}, J=11.2,2.4 \mathrm{~Hz}, 1 \mathrm{H})$, $7.26(\mathrm{~m}, 3 \mathrm{H}), 7.21-7.11(\mathrm{~m}, 3 \mathrm{H}), 6.75(\mathrm{t}, J=4.8 \mathrm{~Hz}, 1 \mathrm{H}), 6.55(\mathrm{td}$, $J=8.7,2.5 \mathrm{~Hz}, 1 \mathrm{H}), 5.70(\mathrm{~s}, 1 \mathrm{H}), 5.13(\mathrm{~d}, J=6.6 \mathrm{~Hz}, 1 \mathrm{H}), 3.39$ (d, $J$ $=6.6 \mathrm{~Hz}, 1 \mathrm{H}), 2.10(\mathrm{~s}, 3 \mathrm{H}), 1.97(\mathrm{~s}, 3 \mathrm{H}) .{ }^{13} \mathrm{C} \mathrm{NMR}(151 \mathrm{MHz}$, $\left.\mathrm{CDCl}_{3}\right) \delta 193.9,163.4,161.8,159.5,157.8,147.7,144.9,141.7$,
128.7 , 126.7, 126.2, 125.1, 121.4, 113.2, 111.1, 107.9, 107.8, 103.9, 103.8, 52.6, 35.1, 28.8, 27.1, 14.1. ${ }^{19} \mathrm{~F}$ NMR (376 MHz, $\left.\mathrm{CDCl}_{3}\right) \delta-113.52(1 \mathrm{~F})$. HRMS calculated for $\mathrm{C}_{26} \mathrm{H}_{21} \mathrm{FN}_{3} \mathrm{O}_{2}+(\mathrm{M}+$ H) ${ }^{+}$: 426.1612; observed: 426.1615 .

3ua was obtained according to the general procedure, with $88 \%$ yield, $\mathrm{dr}>20: 1$; pale yellow solid. ${ }^{1} \mathrm{H}$ NMR $(600 \mathrm{MHz}$, $\left.\mathrm{CDCl}_{3}\right) \delta 8.54(\mathrm{~s}, 2 \mathrm{H}), 7.35(\mathrm{t}, J=7.2 \mathrm{~Hz}, 2 \mathrm{H}), 7.27(\mathrm{t}, J=7.7 \mathrm{~Hz}$, $3 \mathrm{H}), 7.14(\mathrm{~d}, J=7.2 \mathrm{~Hz}, 1 \mathrm{H}), 6.99(\mathrm{t}, J=7.6 \mathrm{~Hz}, 1 \mathrm{H}), 6.87(\mathrm{~s}, 1 \mathrm{H})$, $6.79(\mathrm{~d}, J=8.0 \mathrm{~Hz}, 1 \mathrm{H}), 6.16(\mathrm{~s}, 1 \mathrm{H}), 5.12(\mathrm{~d}, J=6.5 \mathrm{~Hz}, 1 \mathrm{H}), 3.68$ (s, 3H), 3.57 (d, $J=6.5 \mathrm{~Hz}, 1 \mathrm{H}), 2.26(\mathrm{~s}, 3 \mathrm{H}), 2.17(\mathrm{~s}, 3 \mathrm{H}) .{ }^{13} \mathrm{C}$ NMR $\left(151 \mathrm{MHz}, \mathrm{CDCl}_{3}\right) \delta 193.9,159.5,157.9,157.7,147.6$, 144.9, 141.6, 129.7, 129.7, 126.8, 125.8, 124.3, 121.4, 121.1, $118.7,113.2,111.1,52.2,35.3,28.9,27.0,14.1$. HRMS calculated for $\mathrm{C}_{27} \mathrm{H}_{24} \mathrm{~N}_{3} \mathrm{O}_{3}{ }^{+}(\mathrm{M}+\mathrm{H})^{+}:$: 438.1812; observed: 438.1819 .

3va was obtained according to the general procedure, with $90 \%$ yield, $\mathrm{dr}>20: 1$; pale yellow solid. ${ }^{1} \mathrm{H}$ NMR $(400 \mathrm{MHz}$, $\left.\mathrm{CDCl}_{3}\right) \delta 7.97(\mathrm{~d}, J=8.2 \mathrm{~Hz}, 1 \mathrm{H}), 7.30(\mathrm{~m}, 4 \mathrm{H}), 7.19(\mathrm{~d}, J=3.2 \mathrm{~Hz}$, $1 \mathrm{H}), 7.05(\mathrm{td}, J=8.0,1.4 \mathrm{~Hz}, 1 \mathrm{H}), 7.00(\mathrm{dd}, J=5.3,3.4 \mathrm{~Hz}, 2 \mathrm{H})$, $6.94(\mathrm{td}, J=7.4,1.0 \mathrm{~Hz}, 1 \mathrm{H}), 5.77(\mathrm{~s}, 1 \mathrm{H}), 4.77(\mathrm{~d}, J=6.8 \mathrm{~Hz}, 1 \mathrm{H})$, $3.56(\mathrm{~d}, J=6.8 \mathrm{~Hz}, 1 \mathrm{H}), 2.30(\mathrm{~s}, 3 \mathrm{H}), 2.05(\mathrm{~s}, 3 \mathrm{H}), 1.39(\mathrm{~s}, 9 \mathrm{H}) .{ }^{13} \mathrm{C}$ NMR (151 MHz, $\left.\mathrm{CDCl}_{3}\right) \delta 193.9,177.2,157.8,146.7,145.4$, $141.1,130.1,129.2,128.8,128.1,128.0,126.8,125.4,124.4$, 123.5, 121.4, 117.9, 112.2, 52.6, 40.7, 37.8, 28.9, 28.1, 27.8, 14.2. HRMS calculated for $\mathrm{C}_{27} \mathrm{H}_{24} \mathrm{~N}_{3} \mathrm{O}_{3}{ }^{+}(\mathrm{M}+\mathrm{H})^{+}$: 438.1812; observed: 438.1819 .

3ab was obtained according to the general procedure, with $66 \%$ yield, $\mathrm{dr}>20: 1$; pale yellow solid. ${ }^{1} \mathrm{H}$ NMR $(400 \mathrm{MHz}$, $\left.\mathrm{CDCl}_{3}\right) \delta 8.53(\mathrm{~d}, J=4.7 \mathrm{~Hz}, 2 \mathrm{H}), 8.10(\mathrm{~d}, J=8.0 \mathrm{~Hz}, 1 \mathrm{H}), 7.78(\mathrm{td}$, $J=7.6,1.8 \mathrm{~Hz}, 1 \mathrm{H}), 7.44(\mathrm{dd}, J=7.3,0.8 \mathrm{~Hz}, 1 \mathrm{H}), 7.24-7.17(\mathrm{~m}$, 1H), 7.15-7.06 (m, 2H), 7.00 (ddd, $J=10.1,8.2,1.2 \mathrm{~Hz}, 1 \mathrm{H}$ ), 6.90 (td, $J=7.4,1.0 \mathrm{~Hz}, 1 \mathrm{H}), 6.75(\mathrm{t}, J=4.8 \mathrm{~Hz}, 1 \mathrm{H}), 5.64(\mathrm{~s}, 1 \mathrm{H}), 5.19$ (d, $J=6.7 \mathrm{~Hz}, 1 \mathrm{H}$ ), 3.47 (d, $J=6.7 \mathrm{~Hz}, 1 \mathrm{H}), 2.02$ (s, 3H), 1.93 (s, 3H). ${ }^{13} \mathrm{C}$ NMR (151 MHz, $\left.\mathrm{CDCl}_{3}\right) \delta 194.06$ (s), 162.9, 161.3, $160.0,157.6,157.3,147.9,143.9,132.2$ (d, $J=3.4 \mathrm{~Hz}$ ), 130.0, $129.4(\mathrm{~d}, J=8.3 \mathrm{~Hz}), 127.8$ (d, $J=13.2 \mathrm{~Hz}), 127.7,125.1,124.4$ (d, $J=3.6 \mathrm{~Hz}), 121.6,121.2,116.1$ (d, $J=21.2 \mathrm{~Hz}), 115.6,112.6$, 110.0, 50.1, 33.1, 28.8, 23.7, 13.9. ${ }^{19} \mathrm{~F}$ NMR (376 $\left.\mathrm{MHz} \mathrm{CDCl}_{3}\right) \delta$ -114.19 (1F). HRMS calculated for HRMS calculated for $\mathrm{C}_{26} \mathrm{H}_{21} \mathrm{FN}_{3} \mathrm{O}_{2}+(\mathrm{M}+\mathrm{H})^{+}:$: 426.1612; observed: 426.1612 .

3ac was obtained according to the general procedure, with $74 \%$ yield, $\mathrm{dr}>20: 1$; pale yellow solid. ${ }^{1} \mathrm{H}$ NMR $(600 \mathrm{MHz}$, $\left.\mathrm{CDCl}_{3}\right) \delta 8.55(\mathrm{~d}, J=4.7 \mathrm{~Hz}, 2 \mathrm{H}), 8.17(\mathrm{t}, J=7.0 \mathrm{~Hz}, 1 \mathrm{H}), 7.43(\mathrm{~d}, J$ $=7.3 \mathrm{~Hz}, 1 \mathrm{H}), 7.30-7.19(\mathrm{~m}, 1 \mathrm{H}), 7.14(\mathrm{t}, J=7.8 \mathrm{~Hz}, 1 \mathrm{H})$, $7.10-7.03(\mathrm{~m}, 3 \mathrm{H}), 6.94(\mathrm{t}, J=7.4 \mathrm{~Hz}, 1 \mathrm{H}), 6.78(\mathrm{t}, J=4.7 \mathrm{~Hz}$, 1H), $5.74(\mathrm{~d}, J=5.7 \mathrm{~Hz}, 1 \mathrm{H}), 5.20(\mathrm{~d}, J=6.5 \mathrm{~Hz}, 1 \mathrm{H}), 3.50(\mathrm{~d}, J=$ $6.5 \mathrm{~Hz}, 1 \mathrm{H}), 2.36(\mathrm{~s}, 3 \mathrm{H}), 2.16(\mathrm{~s}, 3 \mathrm{H}), 2.02(\mathrm{~s}, 3 \mathrm{H}) .{ }^{13} \mathrm{C}$ NMR $(151$ $\left.\mathrm{MHz} \mathrm{CDCl}_{3}\right) \delta 194.1,159.9,157.7147 .9,143.7,141.9,138.3$, 130.7, 128.6, 127.5, 127.4, 127.3, 124.8, 123.8, 121.5, 121.3, $115.6,112.6,111.1,51.9,35.9,28.8,27.1,21.6,14.0$. HRMS calculated for $\mathrm{C}_{27} \mathrm{H}_{24} \mathrm{~N}_{3} \mathrm{O}_{2}{ }^{+}(\mathrm{M}+\mathrm{H})^{+}$: 422.1863; observed: 422.1869;

3ad was obtained according to the general procedure, with $90 \%$ yield, dr > 20:1; pale yellow solid. ${ }^{1} \mathrm{H}$ NMR $(400 \mathrm{MHz}$, $\left.\mathrm{CDCl}_{3}\right) \delta 8.56(\mathrm{~d}, J=4.7 \mathrm{~Hz}, 2 \mathrm{H}), 8.17(\mathrm{~d}, J=8.2 \mathrm{~Hz}, 1 \mathrm{H}), 7.44(\mathrm{~d}$, $J=7.3 \mathrm{~Hz}, 1 \mathrm{H}$ ), 7.31 (d, $J=8.5 \mathrm{~Hz}, 2 \mathrm{H}), 7.21$ (d, $J=8.5 \mathrm{~Hz}, 2 \mathrm{H}$ ), $7.15(\mathrm{t}, J=7.8 \mathrm{~Hz}, 1 \mathrm{H}), 6.95(\mathrm{t}, J=7.4 \mathrm{~Hz}, 1 \mathrm{H}), 6.81(\mathrm{t}, J=4.7 \mathrm{~Hz}$, $1 \mathrm{H}), 5.73(\mathrm{~s}, 1 \mathrm{H}), 5.16(\mathrm{~d}, J=6.6 \mathrm{~Hz}, 1 \mathrm{H}), 3.49(\mathrm{~d}, J=6.6 \mathrm{~Hz}, 1 \mathrm{H})$, 
2.16 (s, 3H), 2.02 (s, 3H). ${ }^{13} \mathrm{C}$ NMR (151 MHz, $\left.\mathrm{CDCl}_{3}\right) \delta 193.99$, 159.79, 157.89, 157.7, 147.4, 143.7, 140.5, 132.5, 130.2, 128.7, 128.2, 127.7, 124.9, 121.6, 121.3, 115.6, 112.8, 111.2, 51.9, 35.9, 28.8, 26.7, 14.0. HRMS calculated for calculated for $\mathrm{C}_{26} \mathrm{H}_{21} \mathrm{ClN}_{3} \mathrm{O}_{2}+(\mathrm{M}+\mathrm{H})^{+}:$442.1317; observed: 442.1319 .

3ae was obtained according to the general procedure, with $91 \%$ yield, $\mathrm{dr}=15: 1$; pale yellow solid. ${ }^{1} \mathrm{H}$ NMR $(600 \mathrm{MHz}$, $\left.\mathrm{CDCl}_{3}\right) \delta 8.54(\mathrm{~d}, J=4.7 \mathrm{~Hz}, 2 \mathrm{H}), 8.16(\mathrm{~d}, J=8.2 \mathrm{~Hz}, 1 \mathrm{H}), 7.42(\mathrm{~d}$, $J=7.3 \mathrm{~Hz}, 1 \mathrm{H}), 7.18$ (d, $J=8.2 \mathrm{~Hz}, 2 \mathrm{H}), 7.17-7.11(\mathrm{~m}, 3 \mathrm{H}), 6.93$ $(\mathrm{td}, J=7.5,0.8 \mathrm{~Hz}, 1 \mathrm{H}), 6.78(\mathrm{t}, J=4.7 \mathrm{~Hz}, 1 \mathrm{H}), 5.72(\mathrm{~d}, J=2.4 \mathrm{~Hz}$, 1H), $5.17(\mathrm{~d}, J=6.5 \mathrm{~Hz}, 1 \mathrm{H}), 3.48(\mathrm{~d}, J=6.5 \mathrm{~Hz}, 1 \mathrm{H}), 2.33(\mathrm{~s}, 3 \mathrm{H})$, 2.15 (s, 3H), 2.01(s, 3H). ${ }^{13} \mathrm{C}$ NMR (151 MHz, $\left.\mathrm{CDCl}_{3}\right) \delta 194.0$, 159.9, 157.6 (d, $J=2.3 \mathrm{~Hz}$ ), 148.2, 143.8, 138.9, 136.3, 130.6, $129.4,127.5,126.8,124.8,121.5,121.2,115.6,112.6,110.8$, 51.8, 35.6, 28.8, 26.9, 21.0, 14.0. HRMS: calculated for $\mathrm{C}_{27} \mathrm{H}_{24} \mathrm{~N}_{3} \mathrm{O}_{2}+(\mathrm{M}+\mathrm{H})^{+}:$422.1863; observed: 422.1865 .

3af was obtained according to the general procedure, with $76 \%$ yield, $\mathrm{dr}=15: 1$; pale yellow solid. ${ }^{1} \mathrm{H}$ NMR $(600 \mathrm{MHz}$, $\left.\mathrm{CDCl}_{3}\right) \delta 8.46(\mathrm{~d}, J=4.7 \mathrm{~Hz}, 2 \mathrm{H}), 8.08(\mathrm{~d}, J=7.9 \mathrm{~Hz}, 1 \mathrm{H}), 7.34(\mathrm{~d}$, $J=7.3 \mathrm{~Hz}, 1 \mathrm{H}), 7.13-7.08(\mathrm{~m}, 4 \mathrm{H}), 7.05(\mathrm{t}, J=7.8 \mathrm{~Hz}, 1 \mathrm{H}), 6.85$ $(\mathrm{t}, J=7.4 \mathrm{~Hz}, 1 \mathrm{H}), 6.69(\mathrm{t}, J=4.7 \mathrm{~Hz}, 1 \mathrm{H}), 5.65(\mathrm{~d}, J=1.9 \mathrm{~Hz}, 1 \mathrm{H})$, 5.09 (d, $J=6.5 \mathrm{~Hz}, 1 \mathrm{H}$ ), 3.40 (d, $J=6.5 \mathrm{~Hz}, 1 \mathrm{H}$ ), 2.55 (q, $J=7.6$ $\mathrm{Hz}, 2 \mathrm{H}), 2.07$ (s, 3H), 1.93 (s, 3H), $1.14(\mathrm{t}, J=7.6 \mathrm{~Hz}, 3 \mathrm{H}) .{ }^{13} \mathrm{C}$ NMR (151 MHz, $\left.\mathrm{CDCl}_{3}\right) \delta 194.0,159.9,157.77,148.1,143.8$, $142.7,139.2,130.7,128.2,127.5,126.8,124.8,121.5,121.2$, 115.6, 112.6, 110.9, 51.8, 37.2, 28.8, 28.5, 26.9, 15.7, 14.0. HRMS calculated for $\mathrm{C}_{26} \mathrm{H}_{26} \mathrm{~N}_{3} \mathrm{O}_{2}+(\mathrm{M}+\mathrm{H})^{+}:$436.2020; observed: 436.2025 .

3ag was obtained according to the general procedure, with $67 \%$ yield, $\mathrm{dr}=18: 1$; pale yellow solid. ${ }^{1} \mathrm{H}$ NMR $(400 \mathrm{MHz}$, $\left.\mathrm{CDCl}_{3}\right) \delta 8.47(\mathrm{~d}, J=4.8 \mathrm{~Hz}, 2 \mathrm{H}), 8.10(\mathrm{~d}, J=8.2 \mathrm{~Hz}, 1 \mathrm{H}), 7.50(\mathrm{~d}$, $J=8.3 \mathrm{~Hz}, 2 \mathrm{H}), 7.36(\mathrm{dd}, J=7.6,0.8 \mathrm{~Hz}, 1 \mathrm{H}), 7.21(\mathrm{~d}, J=8.2 \mathrm{~Hz}$, $2 \mathrm{H}), 7.08(\mathrm{td}, J=8.1,1.3 \mathrm{~Hz}, 1 \mathrm{H}), 6.87(\mathrm{td}, J=7.4,1.0 \mathrm{~Hz}, 1 \mathrm{H}$ ), $6.73(\mathrm{t}, J=4.8 \mathrm{~Hz}, 1 \mathrm{H}), 5.69(\mathrm{~s}, 1 \mathrm{H}), 5.12(\mathrm{~d}, J=6.6 \mathrm{~Hz}, 1 \mathrm{H}), 3.46$ $(\mathrm{d}, J=6.6 \mathrm{~Hz}, 1 \mathrm{H}), 2.08(\mathrm{~s}, 3 \mathrm{H}), 1.95(\mathrm{~s}, 3 \mathrm{H}) .{ }^{13} \mathrm{C}$ NMR $(151 \mathrm{MHz}$, $\left.\mathrm{CDCl}_{3}\right) \delta 193.8,1597,158.0,157.7,146.8,146.4,143.7,130.1$, 128.7, 128.5, 127.8, 126.7, 125.5 (d, $J=3.7 \mathrm{~Hz}$ ), 124.9, 121.7, 121.3, 115.7, 112.9, 111.8, 52.5, 36.7, 28.8, 26.9, 14.0. ${ }^{19} \mathrm{~F}$ NMR (565 MHz, $\left.\mathrm{CDCl}_{3}\right) \delta-62.39$ (s, 1F). HRMS calculated for $\mathrm{C}_{27} \mathrm{H}_{21} \mathrm{~F}_{3} \mathrm{~N}_{3} \mathrm{O}_{2}+(\mathrm{M}+\mathrm{H})^{+}$: 476.1580; observed: 476.1584 .

3ah was obtained according to the general procedure, with $68 \%$ yield, $\mathrm{dr}>20: 1$; pale yellow solid. ${ }^{1} \mathrm{H}$ NMR $(400 \mathrm{MHz}$, $\left.\mathrm{CDCl}_{3}\right) \delta 8.48(\mathrm{~d}, J=4.7 \mathrm{~Hz}, 2 \mathrm{H}), 8.09(\mathrm{~d}, J=8.2 \mathrm{~Hz}, 1 \mathrm{H}), 7.36(\mathrm{~d}$, $J=7.4 \mathrm{~Hz}, 1 \mathrm{H}), 7.25-7.19(\mathrm{~m}, 2 \mathrm{H}), 7.07$ (t, $J=7.8 \mathrm{~Hz}, 1 \mathrm{H}), 6.96$ $(\mathrm{t}, J=8.7 \mathrm{~Hz}, 2 \mathrm{H}), 6.87(\mathrm{t}, J=7.0 \mathrm{~Hz}, 1 \mathrm{H}), 6.73(\mathrm{t}, J=4.0 \mathrm{~Hz}, 1 \mathrm{H})$, $5.64(\mathrm{~d}, J=1.0 \mathrm{~Hz}, 1 \mathrm{H}), 5.07$ (d, $J=6.6 \mathrm{~Hz}, 1 \mathrm{H}), 3.40$ (d, $J=6.6$ $\mathrm{Hz}, 1 \mathrm{H}), 2.08$ (s, 3H), 1.94 (s, 3H). ${ }^{13} \mathrm{C}$ NMR (151 MHz, $\left.\mathrm{CDCl}_{3}\right) \delta$ 193.9, 162.6, 160.9, 159.8, 157.7 (d, $J=9.3 \mathrm{~Hz}$ ), 147.9, 143.8, $137.5,130.3,128.8$ (d, $J=8.0 \mathrm{~Hz}$ ), 127.6, 124.9, 121.6, 121.3, $115.6(\mathrm{~d}, J=6.7 \mathrm{~Hz}), 115.5,112.7,110.8,51.7$ 35.4, 28.8, 26.8, 14.0. ${ }^{19} \mathrm{~F}$ NMR (376 $\left.\mathrm{MHz}, \mathrm{CDCl}_{3}\right) \delta-115.97$ (1F). HRMS calculated for $\mathrm{C}_{26} \mathrm{H}_{21} \mathrm{FN}_{3} \mathrm{O}_{2}{ }^{+}(\mathrm{M}+\mathrm{H})^{+}$: 426.1612; observed: 426.1613 .

3ai was obtained according to the general procedure, with $67 \%$ yield, $\mathrm{dr}>20: 1$; pale yellow solid. ${ }^{1} \mathrm{H}$ NMR $(400 \mathrm{MHz}$, $\left.\mathrm{CDCl}_{3}\right) \delta 8.47(\mathrm{~d}, J=4.8 \mathrm{~Hz}, 2 \mathrm{H}), 8.09(\mathrm{~d}, J=8.2 \mathrm{~Hz}, 1 \mathrm{H})$, 7.42-7.28 (m, 1H), 7.23 (dd, $J=4.9,3.1 \mathrm{~Hz}, 1 \mathrm{H}), 7.06$ (s, 1H),
6.89-6.82 (m, 1H), $6.72(\mathrm{t}, J=4.8 \mathrm{~Hz}, 3 \mathrm{H}), 5.69(\mathrm{~s}, 1 \mathrm{H}), 5.09(\mathrm{~d}, J$ $=6.5 \mathrm{~Hz}, 1 \mathrm{H}), 3.41(\mathrm{~d}, J=6.5 \mathrm{~Hz}, 1 \mathrm{H}), 2.11(\mathrm{~s}, 3 \mathrm{H}), 1.96(\mathrm{~s}, 3 \mathrm{H})$. ${ }^{13} \mathrm{C}$ NMR $\left(151 \mathrm{MHz}, \mathrm{CDCl}_{3}\right) \delta 194.0,159.7,157.7,147.8,143.5$, $143.2,130.2,127.6,126.0,125.9,124.8,121.6,121.2,119.2$, 115.6, 112.7, 110.9, 52.0, 36.3, 28.8, 24.3, 14.0. HRMS calculated for $\mathrm{C}_{24} \mathrm{H}_{20} \mathrm{~N}_{3} \mathrm{O}_{2} \mathrm{~S}^{+}(\mathrm{M}+\mathrm{H})^{+}$: 414.1271; observed: 414.1273 .

3aj was obtained according to the general procedure, with $85 \%$ yield, $\mathrm{dr}>20: 1$; pale yellow solid. ${ }^{1} \mathrm{H}$ NMR $(400 \mathrm{MHz}$, $\left.\mathrm{CDCl}_{3}\right) \delta 8.41(\mathrm{~d}, J=4.7 \mathrm{~Hz}, 2 \mathrm{H}), 7.97(\mathrm{~d}, J=8.2 \mathrm{~Hz}, 1 \mathrm{H}), 7.13(\mathrm{~s}$, $1 \mathrm{H}), 6.94(\mathrm{~s}, 1 \mathrm{H}), 6.76(\mathrm{~d}, J=7.4 \mathrm{~Hz}, 1 \mathrm{H}), 6.64(\mathrm{~s}, 1 \mathrm{H}), 5.28(\mathrm{~s}$, $1 \mathrm{H}), 4.73(\mathrm{~d}, J=6.0 \mathrm{~Hz}, 1 \mathrm{H}), 3.03(\mathrm{~d}, J=6.0 \mathrm{~Hz}, 1 \mathrm{H}), 1.99(\mathrm{~s}, 3 \mathrm{H})$, $1.87(\mathrm{~s}, 3 \mathrm{H}),-0.00(\mathrm{~s}, 9 \mathrm{H}) .{ }^{13} \mathrm{C}$ NMR (151 MHz, $\left.\mathrm{CDCl}_{3}\right) \delta 194.2$, $160.0,157.5,156.7,149.3,142.7,130.7,126.9,124.6,121.3$, 121.2, 115.5, 112.25, 108.7, 47.3, 29.2, 28.8, 14.4, 13.9, -2.9. HRMS calculated for $\mathrm{C}_{23} \mathrm{H}_{26} \mathrm{~N}_{3} \mathrm{O}_{2} \mathrm{Si}^{+}(\mathrm{M}+\mathrm{H})^{+}$: 404.1789; observed: 404.1784 .

3ak was obtained according to the general procedure, with $38 \%$ yield, $\mathrm{dr}=10: 1$; pale yellow solid. ${ }^{1} \mathrm{H}$ NMR $(400 \mathrm{MHz}$, $\left.\mathrm{CDCl}_{3}\right) \delta 8.46(\mathrm{~d}, J=4.8 \mathrm{~Hz}, 2 \mathrm{H}), 8.05(\mathrm{~d}, J=8.2 \mathrm{~Hz}, 1 \mathrm{H}), 7.27(\mathrm{~d}$, $J=7.4 \mathrm{~Hz}, 1 \mathrm{H}), 7.02(\mathrm{t}, J=7.8 \mathrm{~Hz}, 1 \mathrm{H}), 6.82(\mathrm{t}, J=7.4 \mathrm{~Hz}, 1 \mathrm{H})$, $6.69(\mathrm{t}, J=4.8 \mathrm{~Hz}, 1 \mathrm{H}), 5.46(\mathrm{~s}, 1 \mathrm{H}), 4.62(\mathrm{~d}, J=6.5 \mathrm{~Hz}, 1 \mathrm{H}), 2.90$ (d, $J=6.5 \mathrm{~Hz}, 1 \mathrm{H}), 2.13(\mathrm{~s}, 3 \mathrm{H}), 1.94(\mathrm{~s}, 3 \mathrm{H}), 1.76-1.62(\mathrm{~m}, 1 \mathrm{H})$, 1.59-1.49 (m, 1H), 1.34 (m, 4H), 0.83 (M, 3H). ${ }^{13} \mathrm{C}$ NMR (151 $\left.\mathrm{MHz}, \mathrm{CDCl}_{3}\right) \delta 194.2,159.9,157.6,157.1,148.9,143.7,130.6$, 127.1, 124.5, 121.3, 121.1, 115.5, 112.3, 109.8, 49.5, 35.8, 32.2, 28.9, 28.8, 23.9, 22.6, 14.1, 14.0. HRMS calculated for $\mathrm{C}_{24} \mathrm{H}_{26} \mathrm{~N}_{3} \mathrm{O}_{2}+(\mathrm{M}+\mathrm{H})^{+}$: 388.2020; observed: 388.2021.

5 was obtained with $96 \%$ yield, pale yellow solid. ${ }^{1} \mathrm{H}$ NMR $\left(400 \mathrm{MHz}, \mathrm{CDCl}_{3}\right) \delta 8.58(\mathrm{~d}, J=4.7 \mathrm{~Hz}, 2 \mathrm{H}), 8.21(\mathrm{~d}, J=8.5 \mathrm{~Hz}$, $1 \mathrm{H}), 7.70(\mathrm{~d}, J=1.8 \mathrm{~Hz}, 1 \mathrm{H}), 7.59(\mathrm{~d}, J=7.3 \mathrm{~Hz}, 2 \mathrm{H}), 7.43(\mathrm{t}, J=$ $7.7 \mathrm{~Hz}, 2 \mathrm{H}), 7.41-7.33(\mathrm{~m}, 4 \mathrm{H}), 7.32-7.26(\mathrm{~m}, 3 \mathrm{H}), 6.83(\mathrm{t}, J=$ $4.7 \mathrm{~Hz}, 1 \mathrm{H}), 5.79(\mathrm{~s}, 1 \mathrm{H}), 5.25$ (d, $J=6.5 \mathrm{~Hz}, 1 \mathrm{H}), 3.59$ (d, $J=6.5$ $\mathrm{Hz}, 1 \mathrm{H}), 2.15$ (s, 3H), $2.01(\mathrm{~s}, 3 \mathrm{H}) .{ }^{13} \mathrm{C}$ NMR (151 MHz, $\left.\mathrm{CDCl}_{3}\right) \delta$ 194.0, 159.8, 157.8, 157.7, 147.8, 143.1, 141.9, 141.2, 134.8, 131.3, 128.8, 128.7, 126.8, 126.7, 126.7, 126.5, 121.3, 115.7, 112.8, 111.2, 52.1, 35.8, 29.7, 29.3, 27.5, 14.0. HRMS calculated for $\mathrm{C}_{32} \mathrm{H}_{26} \mathrm{~N}_{3} \mathrm{O}_{2}+(\mathrm{M}+\mathrm{H})^{+}$: 484.2020; observed: 484.2027.

\section{Results and discussion}

We initiated our investigation by screening the reaction conditions of the coupling of $N$-pyrimidinylindole (1a) with ene-yne ketone (2a) under Co(III)/Zn(II) catalysis (Table 1). The preliminary experimentation highlighted the effectiveness of the combined $\mathrm{Co}(\mathrm{III}) / \mathrm{Zn}(\mathrm{II})$ catalysis. The control experiments revealed that both Co(III) and Zn(II) were important for this transformation. The omission of Co(III) resulted in trace formation of the desired product (entries 1 and 3), whereas the exclusion of Zn(II) resulted in a lower yield (entries 2 and 4). Note that the transformation proceeded in a highly regioselective manner. Neither Co(III) nor Zn(II) is effective against depression when applied alone (entries 1-4). The solvent was observed to exert a dramatic impact on the efficiency. Notably, dichloromethane was identified as the optimal solvent in terms of both the yield and diastereoselectivity, for the formation of 3aa (entries 5-9). Lowering the reaction temperature further resulted in low efficiency (entry 10), and extending the reaction 
Table 1

Optimization studies. a

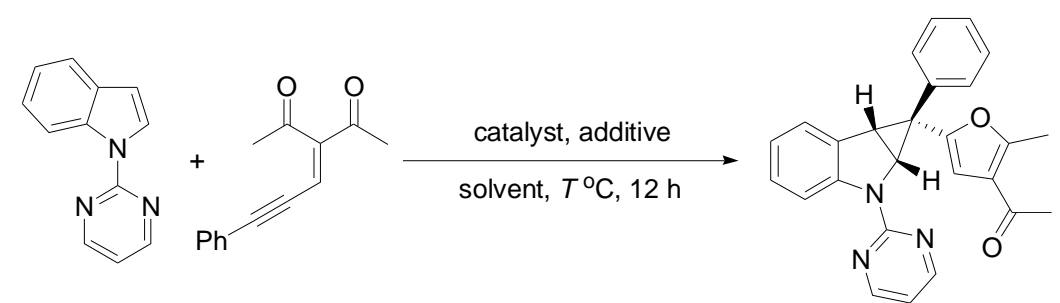

1a

$2 a$

3aa

\begin{tabular}{|c|c|c|c|c|c|c|}
\hline Entry & Catalyst (mol\%) & Solvent $(\mathrm{mL})$ & $T /{ }^{\circ} \mathrm{C}$ & $t / \mathrm{h}$ & Yield ${ }^{\mathrm{b}}(\%)$ & $\mathrm{dr}^{\mathrm{c}}$ \\
\hline 1 & $\mathrm{Zn}(\mathrm{OAc})_{2}(30)$ & TFE (2) & 45 & 12 & trace & - \\
\hline 2 & {$\left[\mathrm{Cp}^{*} \mathrm{Co}(\mathrm{MeCN})_{3}\right]\left[\mathrm{SbF}_{6}\right]_{2}(5)$} & TFE (2) & 45 & 12 & 13 & $15: 1$ \\
\hline 3 & $\mathrm{Zn}(\mathrm{OAc})_{2}(30)$ & DCM (2) & 45 & 12 & 6 & $12: 1$ \\
\hline 4 & {$\left[\mathrm{Cp} * \mathrm{Co}(\mathrm{MeCN})_{3}\right]\left[\mathrm{SbF}_{6}\right]_{2}(5)$} & DCM (2) & 45 & 12 & $34 \%$ & $10: 1$ \\
\hline 5 & {$\left[\mathrm{Cp}^{*} \mathrm{Co}(\mathrm{MeCN})_{3}\right]\left[\mathrm{SbF}_{6}\right]_{2}(5) / \mathrm{Zn}(\mathrm{OAc})_{2}(30)$} & TFE (2) & 45 & 12 & 81 & $14: 1$ \\
\hline 6 & $\left.\mathrm{Cp}^{*} \mathrm{Co}(\mathrm{MeCN})_{3}\right]\left[\mathrm{SbF}_{6}\right]_{2}(5) / \mathrm{Zn}(\mathrm{OAc})_{2}(30)$ & THF (2) & 45 & 12 & 12 & $15: 1$ \\
\hline 7 & $\mathrm{Cp}^{*} \mathrm{Co}\left(\mathrm{MeCN}_{3}\right]\left[\mathrm{SbF}_{6}\right]_{2}(5) / \mathrm{Zn}(\mathrm{OAc})_{2}(30)$ & MeCN (2) & 45 & 12 & 16 & $12: 1$ \\
\hline 8 & $\mathrm{Cp}^{*} \mathrm{Co}\left(\mathrm{MeCN}_{3}\right]\left[\mathrm{SbF}_{6}\right]_{2}(5) / \mathrm{Zn}(\mathrm{OAc})_{2}(30)$ & 1,4-dioxane (2) & 45 & 12 & trace & - \\
\hline 9 & $\left.\mathrm{Cp} * \mathrm{Co}(\mathrm{MeCN})_{3}\right]\left[\mathrm{SbF}_{6}\right]_{2}(5) / \mathrm{Zn}(\mathrm{OAc})_{2}(30)$ & DCM (2) & 45 & 12 & 94 & $>20: 1$ \\
\hline 10 & $\left.\mathrm{Cp}^{*} \mathrm{Co}(\mathrm{MeCN})_{3}\right]\left[\mathrm{SbF}_{6}\right]_{2}(5) / \mathrm{Zn}(\mathrm{OAc})_{2}(30)$ & DCM (2) & 30 & 12 & 86 & $>20: 1$ \\
\hline 11 & $\left.\mathrm{Cp} * \mathrm{Co}(\mathrm{MeCN})_{3}\right]\left[\mathrm{SbF}_{6}\right]_{2}(5) / \mathrm{Zn}(\mathrm{OAc})_{2}(30)$ & DCM (2) & 45 & 24 & 95 & $>20: 1$ \\
\hline
\end{tabular}

a The reaction was carried out using $1 \mathrm{a}(0.2 \mathrm{mmol}), \mathbf{2 a}(0.24 \mathrm{mmol}),\left[\mathrm{Cp}{ }^{*} \mathrm{Co}(\mathrm{MeCN})_{3}\right]\left[\mathrm{SbF}_{6}\right]_{2}(5 \mathrm{~mol} \%)$, and $\mathrm{Zn}(\mathrm{OAc})_{2}(30 \mathrm{~mol} \%)$ in $\mathrm{DCM}(2 \mathrm{~mL})$ at $30-45^{\circ} \mathrm{C}$ for $12-24 \mathrm{~h}$. ${ }^{\mathrm{b}}$ Isolated yield. ${ }^{\mathrm{c}}$ Determined by ${ }^{1} \mathrm{H}$ NMR.

time resulted in a yield (entry 11) approximately equal to that under the optimized reaction conditions (entry 9).

After obtaining the optimized reaction conditions, we investigated the scope of $\mathrm{N}$-pyrimidinyl indoles in the coupling with 2a (Scheme 2). The introduction of substituents with different

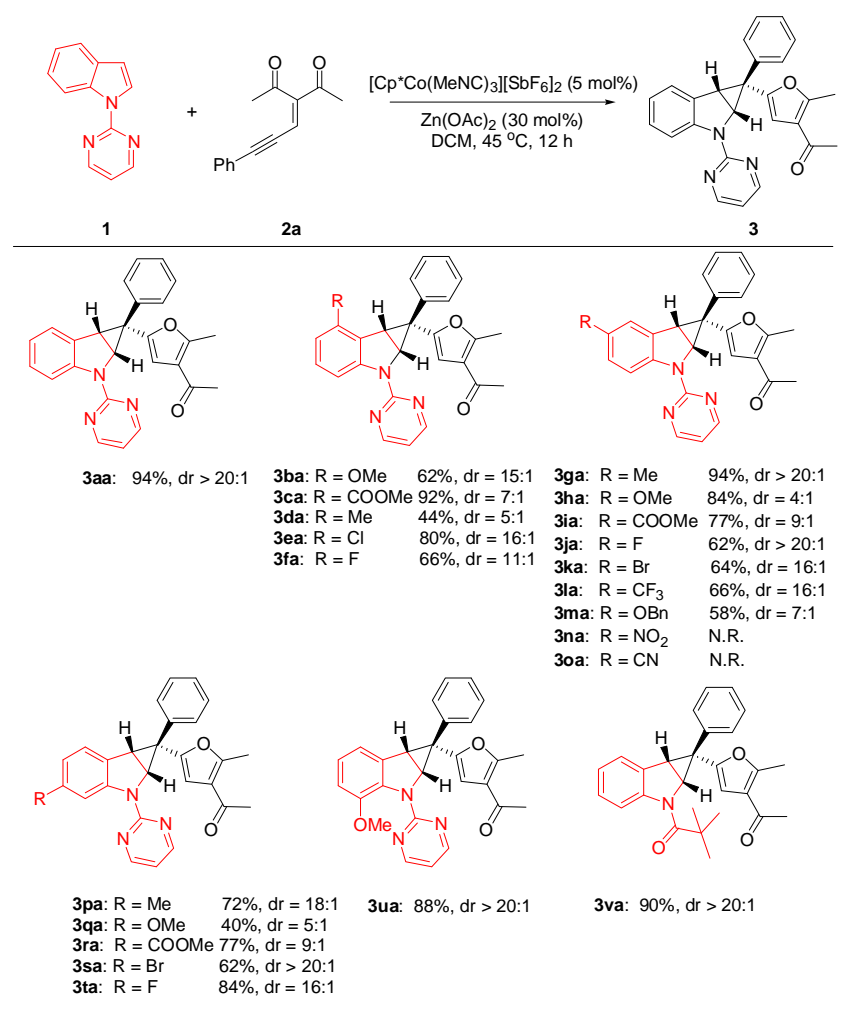

Scheme 2. Scope of indoles. Reaction conditions: 1a (0.2 mmol), 2 (0.24 mmol), $\left[\mathrm{Cp} * \mathrm{Co}(\mathrm{MeCN})_{3}\right]\left[\mathrm{SbF}_{6}\right]_{2}(5 \mathrm{~mol} \%)$, and $\mathrm{Zn}(\mathrm{OAc})_{2}(30 \mathrm{~mol} \%)$ in $\operatorname{DCM}(2 \mathrm{~mL})$ at $45^{\circ} \mathrm{C}$ for $12 \mathrm{~h}$. electronic effects, to different positions of the $\mathrm{N}$-pyrimidinylindole is completely tolerated. With electron-donating groups $(\mathrm{Me}, \mathrm{OMe}$, and $\mathrm{OBn})$, electron-withdrawing groups $\left(\mathrm{CF}_{3}\right.$ and $\left.\mathrm{CO}_{2} \mathrm{Me}\right)$, and halogen $(\mathrm{F}, \mathrm{Cl}$, $\mathrm{Br}$ ) group at the C4-, C5-, or C6-positions of the indole ring, the coupling resulted in the corresponding products at moderate to high yields (3ba-3fa, 3ga-3ma, and 3pa-3ta). The 5- $\mathrm{NO}_{2}$ and 5 -CN indoles failed to yield the desired products. Moreover, a C7-substituted indole is tolerated in the isolation of the products 3ua with $88 \%$ yield. The indole substrates are not limited to $N$-pyrimidinyl indoles; $N$-acyl also proceeded smoothly, and the desired product was obtained with $90 \%$ yield.

Next, the scope of the ene-yne ketones was examined in the coupling with $N$-pyrimidinylindole (Scheme 3). Both the electron-rich and electron-poor ene-yne ketones at the para position could react with 1a to yield the target products 3ad-3ah with reasonable to remarkable yields. Notably, ene-yne ketones bearing methyl (3ab) and fluorine (3ac) at both the ortho and meta positions were established to be viable coupling partners (66\% and 74\% yield, respectively). Heterocycle-based (3ai), TMS (3aj), and alkanes (3ak) substituted substrates coupled smoothly with 1a to produce the desired products with reasonable yields and remarkable diastereoselectivity.

The scale-up ( $5 \mathrm{mmol}$ ) synthesis of 3aa has also been performed, which was isolated with $90 \%$ yield. The derivatization reaction was also examined to demonstrate the utility of this reaction (Scheme 4). The Suzuki cross-coupling reaction of 3sa $(0.25 \mathrm{mmol})$ with benzeneboronic acid $4(0.25 \mathrm{mmol})$ in the presence of $\mathrm{Pd}\left(\mathrm{PPh}_{3}\right)_{4}$ produced product 5 at the $\mathrm{C} 5$ position of the indoline with $96 \%$ yield.

On the basis of our experimental results presented above and literature precedents $[21,45,51,54,55]$, a proposed catalytic 

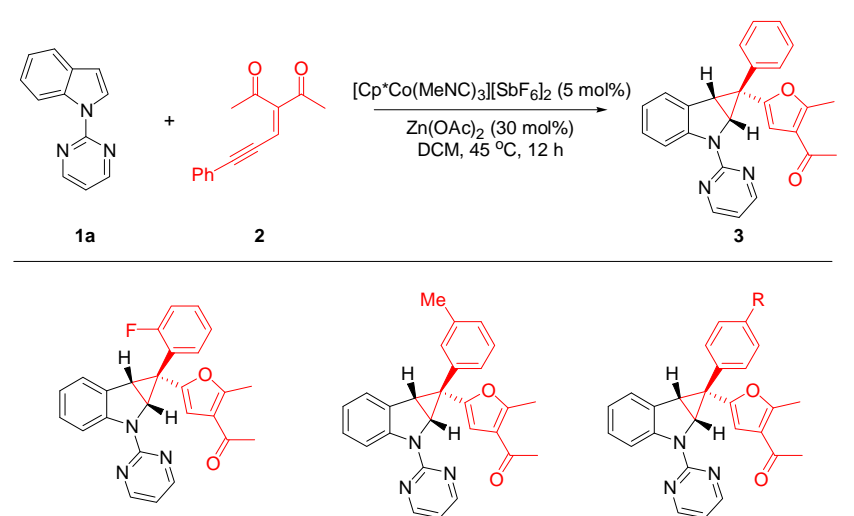

3ad: $\mathrm{R}=\mathrm{Cl} \quad 90 \%, \mathrm{dr}>20: 1$

3ab: $66 \%, d r>20: 1$

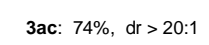

$\begin{array}{ll}\text { 3ae: } R=\text { Me } & 91 \%, \mathrm{dr}=15: 1 \\ \text { 3at } \mathrm{R} & 76 \%, \mathrm{dr}=15: 1\end{array}$

$\begin{array}{ll}\text { 3af: } R=E t & 76 \%, d r=15: 1 \\ \text { 3ag: } R=C_{3} & 67 \%, d r=18: 1 \\ \text { 3ah: } R=F & 68 \%, d r>20: 1\end{array}$

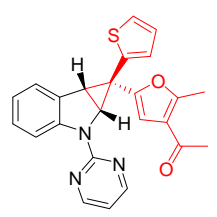

3ai: $67 \%, d r>20: 1$
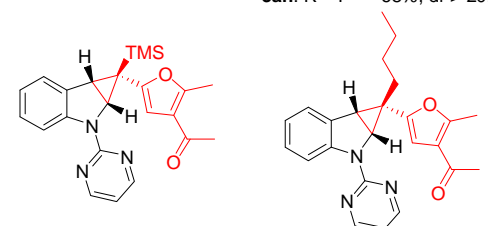

3aj: $85 \%, d r>20: 1$

3ak: $38 \%, \mathrm{dr}=10: 1$

Scheme 3. Scope of ene-yne ketones. Reaction conditions: 1a $(0.2 \mathrm{mmol}), 2(0.24 \mathrm{mmol}),\left[\mathrm{Cp} * \mathrm{Co}(\mathrm{MeCN})_{3}\right]\left[\mathrm{SbF}_{6}\right]_{2}(5 \mathrm{~mol} \%)$, and $\mathrm{Zn}(\mathrm{OAc})_{2}(30$ $\mathrm{mol} \%)$ in DCM $(2 \mathrm{~mL})$ at $45^{\circ} \mathrm{C}$ for $12 \mathrm{~h}$.

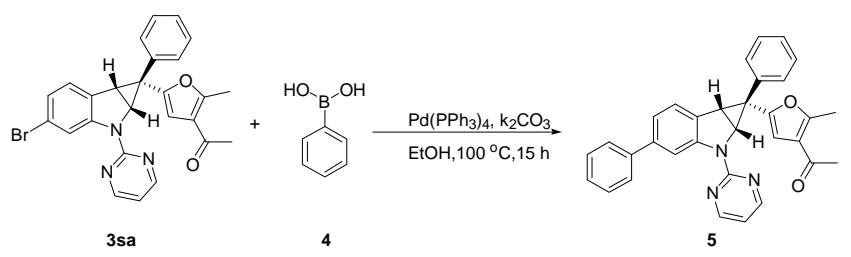

Scheme 4. Derivatization reaction.

cycle is shown in Scheme 5. The electrophilic M-carbene intermediate I is produced by cyclometalation of carbonyl ene-yne with zinc/cobalt salt; then, $\mathrm{Zn}(\mathrm{II}) / \mathrm{Co}(\mathrm{III})$ furylcarbenes is obtained through an intramolecular 5-exo-dig cyclization by the

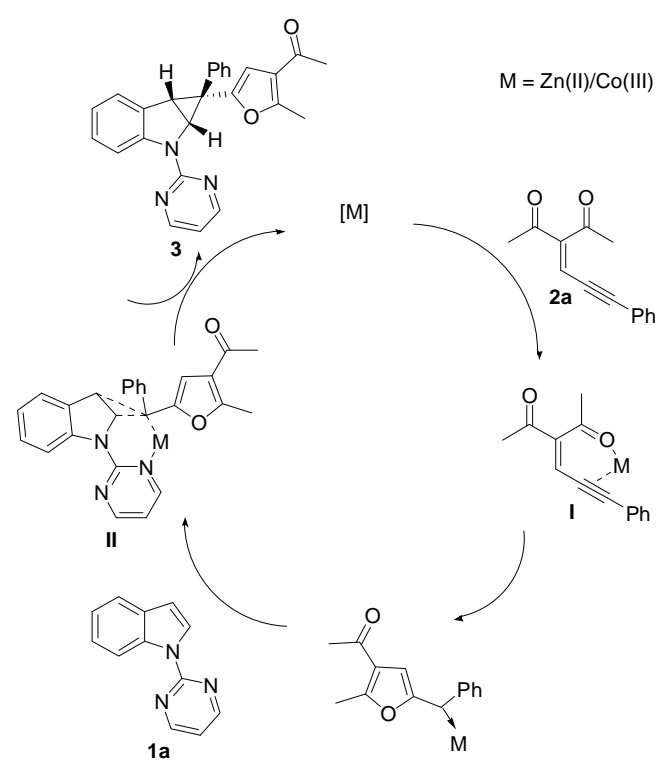

Scheme 5. Proposed mechanism. nucleophilic attack of the carbonyl oxygen atom on the C4 carbon atom of complex I. Subsequent coordinates to indole 1a to give intermediate II, which undergoes cyclization and elimination to form the desired product 3. Further interaction regenerates the active $\mathrm{Zn}(\mathrm{II}) / \mathrm{Co}$ (III) catalyst for the subsequent catalytic cycle.

\section{Conclusions}

We have realized Co(III)/Zn(II)-catalyzed dearomatization of indoles. The reactions are highly efficient and showed diastereoselectivities under mild reaction conditions to produce a series of highly functionalized cyclopropane fused indolines; these indolines are effective for the synthesis of indoline alkaloids. The scope of the protocols was investigated. Considering the mild conditions, broad scope, diastereoselectivities, and high catalytic efficiency, this method is likely to find applications in the synthesis of functionalized-cyclopropane-fused indolines derivatives.

\section{References}

[1] D. Jin, F. Zhang, D. C. Zhang, Chin. J. Org. Chem., 2010, 30, 1005-1009. 


\section{Graphical Abstract}

Chin. J. Catal., 2018, 39: 1881-1889 doi: 10.1016/S1872-2067(18)63154-7

$\mathrm{Co}(\mathrm{III}) / \mathrm{Zn}$ (II)-catalyzed dearomatization of indoles and coupling with carbenes from ene-yne ketones via intramolecular cyclopropanation

Na Li, Junbiao Chang*, Lingheng Kong, Shuangjing Wang, Dandan Wang, Miao Cheng, Xingwei Li*

Henan Normal University;

Xinxiang Medical University;

Dalian Institute of Chemical Physics, Chinese Academy of Sciences

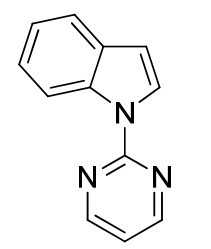<smiles>CC(=O)C(=CC#Cc1ccccc1)C(C)=O</smiles>

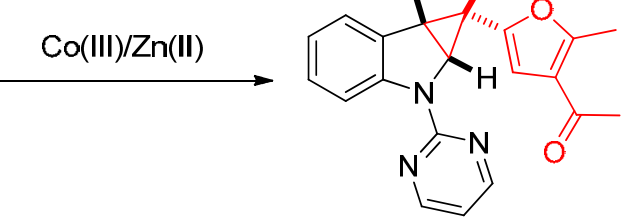

- mild conditions - diastereoselectivity - functionalizable DGs - broad scope (30 examples)

A straightforward and efficient protocol for dearomatizing indoles is described. The reaction, catalyzed by an inexpensive Co(III)/Zn(II) catalyst, starts from conveniently accessible $\mathrm{N}$-pyrimidinyl indoles and ene-yne ketones. Mild reaction conditions, high diastereoselectivity, a broad substrate scope, reasonable functional group tolerance, and reasonable to remarkable yields were observed.

[2] D. Y. Liu, G. W. Zhao, L. Xiang, Eur. J. Org. Chem., 2010, 2010, 3975-3984.

[3] S. Anas, H. B. Kagan, Tetrahedron: Asymmetry, 2009, 20, 2193-2199.

[4] J. Bonjoch, D. Solé, S. García-Rubio, J. Bosch, J. Am. Chem. Soc., 1997, 119, 7230-7240.

[5] D. Zhang, H. Song, Y. Qin, Acc. Chem. Res., 2011, 44, 447-457.

[6] X. Deng, K. J. Liang, X. G. Tong, M. Ding, D. S. Li, C. F. Xia, Org. Lett., 2014, 16, 3276-3279.

[7] T. Wang, Q. G. Xu, P. Yu, X. X. Liu, J. M. Cook, Org. Lett., 2001, 3, 345-348.

[8] X. Y. Fu, J. M. Cook, J. Am. Chem. Soc., 1992, 114, 6910-6912.

[9] J. Li, T. Wang, P. Yu, A. Peterson, R. Weber, D. Soerens, D. Grubisha, D. Bennett, J. M. Cook, J. Am. Chem. Soc., 1999, 121, 6998-7010.

[10] S. J. Jin, J. Gong, Y. Qin, Angew. Chem. Int. Ed., 2015, 54, 2228-2231.

[11] H. X. Huang, S. J. Jin, J. Gong, D. Zhang, H. Song, Y. Qin, Chem. Eur. J., 2015, 21, 13284-13290.

[12] M. S. Kirillova, M. E. Muratore, R. Dorel, A. M. Echavarren, J. Am. Chem. Soc., 2016, 138, 3671-3674.

[13] L. A. Paquette, Chem. Rev., 1986, 86, 733-750.

[14] H. N. C. Wong, M. Y. Hon, C. W. Tse, Y. C. Yip, J. Tanko, T. Hudlicky, Chem. Rev., 1989, 89, 165-198.

[15] O. G. Kulinkovich, Pol. J. Chem., 1997, 71, 849-882.

[16] A. de Meijere, S. I. Kozhushkov, L. P. Hadjiarapoglou, Top. Curr. Chem., 2000, 207, 149-227.

[17] H. Lebel, J. F. Marcoux, C. Molinaro, A. B. Charetter, Chem. Rev., 2003, 103, 977-1050.

[18] D. Gagnon, C. Spino, J. Org. Chem., 2009, 74, 6035-6041.

[19] H. Song, J. Yang, W. Chen, Y. Qin, Org. Lett., 2006, 8, 6011-6014.

[20] B. He, H. Song, Y. Du, Y. Qin, J. Org. Chem., 2009, 74, 298-304

[21] A. R. Reddy, F. Hao, K. Wu, C. Y. Zhou, C. M. Che, Angew. Chem. Int. Ed., 2016, 55, 1810-1815.

[22] B. Zhang, A. G. H. Wee, Chem. Commun., 2008, 4837-4839.

[23] B. Zhang, A. G. H. Wee, Org. Biomol. Chem., 2012, 10, 4597-4608.

[24] G. Özüduru, T. Schubach, M. M. K. Boysen, Org. Lett., 2012, 14, 4990-4993.
[25] D. Gagnon, C. Spino, J. Org. Chem., 2009, 74, 6035-6041.

[26] W. J. Welstead Jr, H. F. Stauffer Jr, L. F. Sancilio, J. Med. Chem., 1974, $17,544-547$

[27] E. Wenkert, M. E. Alonso, H. E. Gottlieb, E. L. Sanchez, R. Pellicciari, P. Cogolli, J. Org. Chem., 1977, 42, 3945-3949.

[28] H. Keller, E. Langer, H. Lehner, Monatsh. Chem., 1977, 108, 123-131.

[29] F. Gnad, M. Poleschak, O. Reiser, Tetrahedron Lett., 2004, 45, 4277-4280.

[30] X. J. Zhang, S. P. Liu, M. Yan, Chin. J. Chem., 2008, 26, 716-720.

[31] D. Zhang, H. Song, Y. Qin, Acc. Chem. Res., 2011, 44, 447-457.

[32] H. M. L. Davies, J. E. Spangler, Adv. Heterocycl. Chem., 2013, 110, 43-72.

[33] H. M. L. Davies, S. J. Hedley, Chem. Soc. Rev., 2007, 36, 1109-1119.

[34] H. Xu, Y. P. Li, Y. Cai, G. P. Wang, S. F. Zhu, Q. L. Zhou, J. Am. Chem. Soc., 2017, 139, 7697-7700.

[35] W. T. Wu, L. Zhang, S. L. You, Acta Chim. Sinica., 2017, 75, 419-438.

[36] D. C. Wang, M. S. Xie, H. M. Guo, G. R. Qu, M. C. Zhang, S. L. You, Angew. Chem. Int. Ed., 2016, 55, 14111-14115.

[37] W. Du, Q. S. Gu, Z. L. Li, D. Yang, J. Am. Chem. Soc., 2015, 137, 1130-1135.

[38] Y. Y. Zhou, C. Uyeda, Angew. Chem. Int. Ed., 2016, 55, 3171-3175.

[39] H. Rudler, T. Durand-Réville, J. Organomet. Chem., 2001, 617-618, 571-587.

[40] D. Dhanaka, R. Kurodab, C. B. Reese, Tetrahedron Lett., 1987, 28, 1827-1830.

[41] H. E. Simmons, R. D. Smith, J. Am. Chem. Soc., 1958, 80, 5323-5324.

[42] S. R. Goudreau, A. B. Charette, J. Am. Chem. Soc., 2009, 131, 15633-15635.

[43] K. Miki, T. Yokoi, F. Nishino, K. Ohe, S. Uemura, J. Organomet. Chem., 2002, 645, 228-234.

[44] K. Miki, F. Nishino, K. Ohe, S. Uemura, J. Am. Chem. Soc., 2002, 124, 5260-5261.

[45] K. Miki, T. Yokoi, F. Nishino, Y. Kato, Y. Washitake, K. Ohe, S. Uemura, J. Org. Chem., 2004, 69, 1557-1564. 
[46] K. Miki, S. Uemura, K. Ohe, Chem. Lett., 2005, 34, 1068-1072.

[47] R. Vicente, J. Gonzalez, L. Riesgo, J. Gonzalez, L. A. Lopez, Angew. Chem. Int. Ed., 2012, 51, 8063-8067.

[48] C. X. Zhuo, W. Zhang, S. L.You, Angew. Chem. Int. Ed., 2012, 51, 12662-12686.

[49] W. Chen, D. S. Ji, Y. C Luo, Z. Y. Wang, P. F. Xu, Org. Chem. Front., 2018, 5, 1768-1771.

[50] N. Yoshikai, ChemCatChem., 2015, 7, 732-734.

[51] M. Moselage, J. Li, L. Ackermann, ACS Catal., 2016, 6, 498-525.

[52] X. L. Han, C. J. Zhou, X. G. Liu, S. S. Zhang, H. G. Wang, Q. J. Li, Org. Lett., 2017, 19, 6108-6111.

[53] W. Zhang, F. Han, J. Tong, C. Xia, J. Liu, Chin. J. Catal., 2017, 38,
805-812.

[54] F. Wang, Q. Wang, M. Bao, X. W. Li, Chin. J. Catal., 2016, 37, 1423-1430.

[55] Y. Chen, J. V. Ruppol, X. P. Zhang, J. Am. Chem. Soc., 2007, 129, 12074-12075.

[56] W. J. Kerr, D. M. Lindsay, P. K. Owens, M. Reid, T. Tuttle, S. Campos, ACS Catal., 2017, 7, 7182-7186.

[57] L. Ackermann, A.V. Lygin, Org. Lett., 2011, 13, 3332-3335.

[58] M. Nishino, K. Hirano, T. Satoh, M. Miura, Angew. Chem. Int. Ed., 2012, 51, 6993-6997.

[59] N. Li, J. B. Chang, L. H. Kong, X. W. Li, Org. Chem. Front., 2018, 5, 1978-1982.

\title{
$\operatorname{Co}(I I I) / Z n(I I)$ 催化吲哚的分子内环丙烷化
}

\author{
李 娜 ${ }^{\mathrm{a}, \mathrm{b}}$, 常俊标 ${ }^{\mathrm{a},}$, 孔令恒 ${ }^{\mathrm{c}}$, 王栓景 ${ }^{\mathrm{a}}$, 王丹丹 ${ }^{\mathrm{a}}$, 程 沝 ${ }^{\mathrm{a}}$, 李兴伟 ${ }^{\mathrm{a}, \mathrm{c}, \text { \# }}$ \\ a 河南师范大学化学化工学院, 河南省有机功能分子和药物创新重点实验室, 河南新乡453007

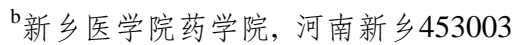 \\ c中国科学院大连化学物理研究所, 辽宁大连 116023
}

\begin{abstract}
摘要: 二氢吲哚骨架被广泛用作不对称合成中的关键结构单元和手性助剂, 也广泛存在于具有多种生物活性的生物碱和 其他天然产物中. 其中2,3位取代的吲哚啉衍生物因其存在的广泛性和生物活性良好而备受关注. 手性环丙烷可通过开环 或环扩展转化为有价值的合成中间体, 构建类似天然产物Lundurines的包含三个季碳立体中心的二氢吲哚并环丙烷结构 是很有吸引力的目标. 传统构建此类化合物的方法包括过渡金属催化和经典的Simmons-Smith反应等. 然而, 更有效、环 境友好和原子经济性的生成卡宾前体的催化方法研究仍然非常有限. 目前由羰基炔化合物和锌盐催化合成多功能性锌-呋 喃卡宾的研究引起了人们的关注. 而 $\mathrm{Cp} * \mathrm{Co}(\mathrm{III})$ 由于其地球丰度、成本效益、低毒性和独特的催化活性已引起越来越多的 注意，但 $\mathrm{Cp} * \mathrm{Co}(\mathrm{III})$ 作为路易斯酸用于催化反应的报道仍然较少.

本文报道了吲哚与烯炔酮通过 $\mathrm{Zn}(\mathrm{II}) / \mathrm{Co}(\mathrm{IIII})$ 呋喃卡宾可以实现分子内环丙烷化, 得到一系列具有三维环状结构的二 氢吲哚化合物. 研究从 $\mathrm{Co}(\mathrm{III}) / \mathrm{Zn}(\mathrm{II})$ 催化 $N$-嘧啶吲哚与烯炔酮偶联反应开始, 条件篎选实验验证了 $\mathrm{Co}(\mathrm{III}) / \mathrm{Zn}(\mathrm{II})$ 催化联合 使用的强大功能. 反应体系中不加 $\mathrm{Co}(\mathrm{III})$ 导致目标产物的痕量形成, 而排除 $\mathrm{Zn}(\mathrm{II})$ 会使收率降低. 本文共完成了 30 个不同 官能团取代的二氢吲哚并环丙烷骨架结构的合成, 目标产物收率从中等到良好, 最高收率可达 $94 \%$. 反应有较好的普适 性, 吲哚基底物不局限于 $N$-吡啶, $N$-酰基反应也进行得很顺利, 达到 $90 \%$ 的收率. 为了提高反应的实用性, 我们进行了放 大实验. 结果表明, 当嘧啶吲哚用量由 0.2 增大至 $5 \mathrm{mmol}$ 时, 反应仍能以较高的收率 $(90 \%)$ 得到目标产物. 此外, 目标产物 还可以进一步衍生转化为其他杂环类化合物, 如在 $\mathrm{Pd}\left(\mathrm{PPh}_{3}\right)_{4}$ 作用下发生Suzuki偶联反应.

总之, 我们在 $\mathrm{Co}(\mathrm{III}) / \mathrm{Zn}(\mathrm{II})$ 催化下成功实现了吲哚的分子内环丙烷化, 合成了一系列含有三个季碳立体中心的二氢吲 哚并环丙烷化合物, 为新药开发奠定了基础. 该催化体系反应条件温和, 底物适用范围广, 非对映体选择性高, 催化效率 高.
\end{abstract}

关键词: Co(III)/Zn(II)催化; 去芳构化; 环丙烷化; 卡宾; 吲哚

收稿日期: 2018-06-21. 接受日期: 2018-07-26. 出版日期: 2018-12-05.

*通讯联系人. 电话: (0411)84379089; 电子信箱: changjunbiao@zzu.edu.cn

\#通讯联系人. 电话: (0411)84379089; 电子信箱: xwli@dicp.ac.cn

基金来源：国家自然科学基金(21525208, 21472186); 河南师范大学研究基金(5101034011009).

本文的电子版全文由Elsevier出版社在ScienceDirect上出版(http://www.sciencedirect.com/science/journal/18722067). 